UMD-PP-09-037

\title{
Vectorlike Confinement at the LHC
}

\author{
Can Kilic ${ }^{a, 1}$, Takemichi Okui ${ }^{a, b, 2}$, and Raman Sundrum ${ }^{a, 3}$ \\ ${ }^{a}$ Department of Physics and Astronomy, Johns Hopkins University, \\ Baltimore, MD 21218, USA \\ ${ }^{b}$ Department of Physics, University of Maryland, \\ College Park, MD 20742, USA
}

\begin{abstract}
We argue for the plausibility of a broad class of vectorlike confining gauge theories at the $\mathrm{TeV}$ scale which interact with the Standard Model predominantly via gauge interactions. These theories have a rich phenomenology at the LHC if confinement occurs at the TeV scale, while ensuring negligible impact on precision electroweak and flavor observables. Spin-1 bound states can be resonantly produced via their mixing with Standard Model gauge bosons. The resonances promptly decay to pseudo-Goldstone bosons, some of which promptly decay to a pair of Standard Model gauge bosons, while others are charged and stable on collider time scales. The diverse set of final states with little background include multiple photons and leptons, missing energy, massive stable charged particles and the possibility of highly displaced vertices in dilepton, leptoquark or diquark decays. Among others, a novel experimental signature of resonance reconstruction out of massive stable charged particles is highlighted. Some of the long-lived states also constitute Dark Matter candidates.
\end{abstract}

${ }^{1}$ kilic@pha.jhu.edu

2 okui@pha.jhu.edu

3 sundrum@pha.jhu.edu 


\section{Contents}

1 Introduction $\quad 2$

2 The Microscopic Structure of Vectorlike Confinement 5

2.1 Hypercolor and Hyperflavor . . . . . . . . . . . . . . . .

2.2 SM Gauge Interactions and the Concept of "Species" . . . . . . . . . . . . . . 8

2.2.1 Mixing of Vector Mesons with SM Gauge Bosons . . . . . . . . . . . . . . 8

2.2.2 Accidental "Species Symmetries" and Long-lived Hyperpions . . . . . . . . . 9

2.3 Nonrenormalizable Interactions . . . . . . . . . . . . . . . . . 10

2.3.1 Mixed SM-hyper 4-fermion Interactions and Hyperpion Decays . . . . . . . . 10

2.3.2 Hyperbaryon Decay . . . . . . . . . . . . . . . . . 10

3 Phenomenological Lagrangian for the LHC 11

3.1 Production and Decay of $\tilde{\rho} \ldots \ldots \ldots \ldots \ldots \ldots \ldots$

3.2 Sources of Hyperpion Masses . . . . . . . . . . . . . . . . . . . . . . . . . . . . . . . .

3.3 Decays of Short-lived Hyperpions . . . . . . . . . . . . . . . . . . . . . . . . . . . . . . . . . . . . .

3.4 Decays of Long-lived Hyperpions . . . . . . . . . . . . . . . . . . . . 14

3.5 Typical Constraints . . . . . . . . . . . . . . . . . . . . . . . . . . . . . . . . . . .

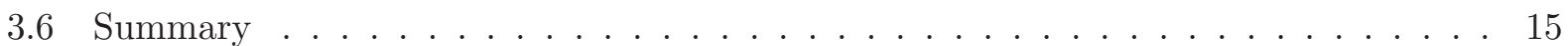

4 Phenomenological Highlights $\quad 16$

4.1 Multiple Gauge-boson Production . . . . . . . . . . . . . . . . 17

4.2 A Model of multi EW-boson Production and Di-CHAMP Resonances . . . . . . . 17

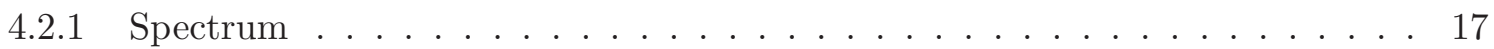

4.2 .2 Model specific constraints . . . . . . . . . . . . . . . . . 18

4.2.3 Decays and collider phenomenology . . . . . . . . . . . . . . . . . . . . . . . . . . .

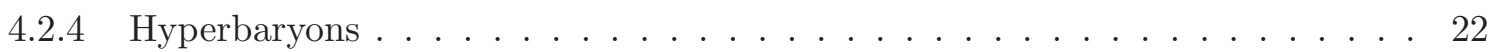

4.3 A Model of Di-R-hadron Resonances . . . . . . . . . . . . . . . . 22

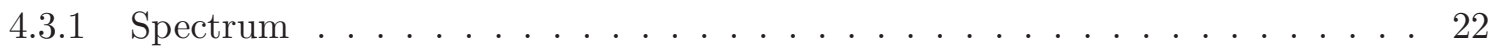

4.3.2 Model specific constraints . . . . . . . . . . . . . . . . . . . . . . . . . . . . . . . . . . .

4.3.3 Decays and collider phenomenology . . . . . . . . . . . . 23

4.3 .4 Hyperbaryons . . . . . . . . . . . . . . . . . . . . . . . . . . . . . . . . .

4.4 Leptoquarks, Dileptons, and Diquarks (with Displaced Vertices) . . . . . . . . 25

4.5 Dark Matter, SUSY Look-alikes and Consistency with Unification . . . . . . . . . 25

5 Summary and Conclusions $\quad 27$

\begin{tabular}{ll} 
A A 2-flavor Model & 28 \\
\hline
\end{tabular}

\begin{tabular}{ll} 
B Collider Constraints on Axion-like $\tilde{\pi}$ & 29 \\
\hline
\end{tabular} 


\section{Introduction}

Expectations for new physics at the LHC have been greatly influenced by the Hierarchy Problem of electroweak symmetry breaking. However, all proposed resolutions of this problem are strongly constrained by existing data, either through the many direct searches for new physics or through virtual sensitivity to new physics in a variety of precision tests. This has led to three operational possibilities: (i) There exists a fully satisfactory solution to the Hierarchy Problem which naturally evades the host of experimental constraints, although we have not discovered it yet. (ii) Naturalness, as usually interpreted, is not a useful or valid principle of particle physics. (iii) Naturalness is an important principle in particle physics, but it is not perfectly satisfied in Nature, there being some modest tuning in the electroweak symmetry breaking sector. That is, the new physics associated with cutting off quadratic divergences in the Standard Model (SM) is heavy enough to naturally evade existing experimental constraints, but possibly at the cost of being out of reach of the LHC!

In either of cases (ii) or (iii), the LHC may still discover new physics, but not directly related to the resolution of the Hierarchy Problem. In case (ii), this would require a coincidence that the new physics just happens to lie within the decade in energy improvement of the LHC over the Tevatron. In case (iii), the odds are better: there may well be some LHC-accessible "low-energy" tail of the spectrum of new physics that cuts off quadratic divergences, the central parts of the spectrum however being somewhat above the reach of the LHC. In cases (ii) and (iii), considerations other than the hierarchy problem are needed to constrain the form of LHC physics to anticipate. Split Supersymmetry [1] is a well known example of either (ii) or (iii), depending on whether the scalar sparticles are orders of magnitude heavier than the TeV scale or just modestly heavier, respectively.

The direction taken in this paper is motivated especially by possibility (iii). From this viewpoint, one of the gauge-field-theoretically simplest and most plausible extensions of the SM is obtained by adding new fermions in vectorlike representations of the SM gauge groups, with a mass scale within the reach of the LHC. One can easily imagine such fermions as remnants of more involved physics at even higher energies. In contrast, adding new chiral fermion representations of the SM gauge group is trickier, because it requires considerable particle content for anomaly cancellation, and because mass generation via electroweak symmetry breaking generically leads to significant oblique corrections to precision electroweak observables. (However, see Ref. 2] for discussion of the 4th generation model and viable regions of parameter space.) The advantage of a vectorlike new fermion, on the other hand, is that it can have mass without coupling to electroweak symmetry breaking so that its impact on precision electroweak observables can be seen to be harmless from the outset.

The new fermions may also interact via new gauge forces, which may be weakly or strongly coupled. While the case of purely weakly coupled new vectorlike fermions is plausible and interesting in its own right, its collider phenomenology is straightforward and we do not pursue this possibility here. Numerous models containing $Z^{\prime}$ s fall in this category.

Instead, we will consider the case where, in addition to SM gauge forces, the new fermions also feel a new strong gauge force that confines at TeV energies, which drastically alters the phenomenology. Calling the new gauge force "hypercolor" 1 the LHC phenomenology will be dominated by

\footnotetext{
${ }^{1}$ The term hypercolor was originally used in 3] to describe what ultimately became known as technicolor-style models. As that use has become extinct, we are employing the term in a new way.
} 
production of the confined "hyperhadrons" made from the vectorlike fermions. There may also exist very weak nonrenormalizable interactions arising from unspecified physics far above the TeV scale, which will only be experimentally relevant at the LHC in mediating the decays of hyperhadrons that cannot decay via hypercolor and SM interactions alone. We will call this scenario "Vectorlike Confinement".

Vectorlike confinement represents a broad category of possible new physics accessible to the LHC, which might represent our first glimpse of an even richer structure at even higher energies (possibly tied to the hierarchy problem). It has a number of attractive features from the viewpoints of both theory and experiment, as well as prospects for rich LHC phenomenology:

- There is a close precedent in familiar particle physics. Particle physics at low energies, below $100 \mathrm{MeV}$ say, is dominated by QED, a precise theory which is beautifully tested. Let us consider this as the analogue of the entire SM tested up to the $\mathrm{TeV}$ scale, the electromagnetic force being the analogue of all the SM gauge forces. We can think of $e^{+} e^{-}$collisions at the $\mathrm{GeV}$ scale as the analogue of turning on the LHC. On the one hand, for no very good reason (as far as we know) the collisions will pair-produce muons, a "new" fermion with vectorlike quantum numbers under the electromagnetic force. This is the analogue of finding new vectorlike fermions at the LHC, but without any new gauge forces involved at these energies. As mentioned above, this is a straightforward possibility, but not what we will focus on.

On the other hand, we can also pair-produce the first generation quarks, also vectorlike with respect to electromagnetism, but feeling an additional strong gauge force, QCD. Quark confinement dominates the phenomenology, quark pair creation being replaced by pion pair production as well as the $\rho$ resonance. The fundamental scale is provided by the confinement scale $\sim$ hundreds of MeV 2 This is the analogue of the Vectorlike Confinement scenario we are proposing; as we will see the pseudo-Goldstone bosons in Vectorlike Confinement will also play a significant role in LHC phenomenology.

While some of the hadrons produced in the $e^{+} e^{-}$collisions decay via electromagnetic interactions alone, for example $\pi^{0} \rightarrow \gamma \gamma$, others such as $\pi^{ \pm}$cannot decay via QCD or QED. However, the weak interactions living at $100 \mathrm{GeV}$, well above the $e^{+} e^{-}$collision energies and the confinement scale, mediate decays of some hadrons, e.g. $\pi^{-} \rightarrow \mu^{-}+\nu^{\prime}$ 's, so that they are merely long-lived rather than absolutely stable. Other hadrons, e.g. the proton, can be essentially stable. Analogous mechanisms give rise to a hierarchy of decay lifetimes in the Vectorlike Confinement scenario as well.

- In some sense, there is a tension between hopes for LHC physics and all past experimental data. If there is exciting new physics at LHC energies, how have its virtual effects remained so well hidden from the myriad precision experiments we have already done? Vectorlike Confinement accomplishes this in a very simple way. TeV-scale confinement can indeed lead to a very rich spectrum of accessible hyperhadron physics, as we shall discuss below. But because the dominant bridge between the SM and the new physics is provided by the SM gauge interactions, the new physics is flavor blind, which allows it to evade the host of constraints from flavor experiments. Furthermore, the new physics, being vectorlike, can be naturally separated from

\footnotetext{
${ }^{2}$ The pions, since they are light pseudo-Goldstone bosons, have masses which are sensitive to the much smaller "current" quark masses.
} 
the electroweak breaking sector (e.g. masses can be set by the confinement scale), thereby evading the body of precision electroweak tests. (Other classes of experimental data will be discussed below.)

- While Vectorlike Confinement involves very modest additions to the SM, as measured by either fundamental particle content or complexity of Lagrangian, it can naturally give rise to a remarkable array of distinct experimental behaviors, including di-gauge-boson resonances, long-lived charged and/or colored states, SUSY look-alike spectra, WIMP dark matter candidates, and leptoquarks. Many of these signals have appeared previously as parts of other scenarios, but often within rather exceptional models or models which are very tightly constrained already by experiments. Here we show how such signals can arise in Vectorlike Confinement models which have very few parameters and need not be tuned to avoid exclusion. We will also see that there are some qualitatively distinct signals that have not been discussed before, such as pair production of collider stable particles through a resonance.

This paper builds on our earlier proposal of new confining vectorlike fermions charged under QCD, presented in [4, which was however restricted in that the new fermions were electroweak singlets 3 Here, we generalize to cases where all SM gauge forces come into play. A theoretically similar class of models with significantly different phenomenology was provided in the "Quirks" model of [6]. These models contain new vectorlike fermions with $\mathrm{TeV}$ scale current masses and extremely low confinement scales. Another closely related class of models is the "Hidden Valley" scenario [7]. While in Vectorlike Confinement the SM gauge interactions provide the bridge to the new physics, in hidden valley models this is done by new interactions ( $Z^{\prime}$ exchange) since the new matter sector is neutral under the SM gauge interactions.

The fact that Vectorlike Confinement involves a new TeV-scale confining force may give the impression that it is a part of the Technicolor scenario and phenomenology [8] (for reviews see [9-12]). But in fact there are very significant differences in motivation, theoretical structure, and experimental implications. Since Technicolor aims at solving the hierarchy problem immediately at the $\mathrm{TeV}$ scale, technifermions are in a chiral representation of electroweak symmetry, which is broken by the $\mathrm{TeV}$ confining force. This leads to an impact on electroweak precision observables which poses a challenge for the Technicolor program. Furthermore Technicolor must be closely tied to flavor physics in order to generate SM fermion masses after electroweak symmetry breaking 4 . In contrast, Vectorlike Confinement does not break electroweak symmetry, thus having negligible impact on electroweak precision observables (electroweak symmetry can be broken by a more or less standard Higgs sector, separated from the new physics). Moreover, Vectorlike Confinement is flavor blind by construction as noted above. We do not however exclude the possibility that Vectorlike Confinement can be a remnant of some even higher scale physics that ameliorates the hierarchy problem using strong dynamics. For comparisons with studies of Technicolor phenomenology see [10,11, 15.

Calculationally, we do borrow the central trick of Technicolor analysis. While we know that confinement, already realized in nature, is a rather typical behavior for non-abelian gauge field theories with qualitatively interesting consequences, the associated strong coupling makes it a no-

\footnotetext{
${ }^{3}$ The Tevatron prospects for discovery in the multijet channel were illustrated in 4 and a follow-up study for the LHC was presented in 5 .

${ }^{4}$ Walking Technicolor [13] and Conformal Technicolor [14] are scenarios which attempt to decouple flavor physics to the far UV. For discussions of similarities and differences between Walking Technicolor and Conformal Technicolor, see Refs. 14.
} 
(Vectorlike Confinement)

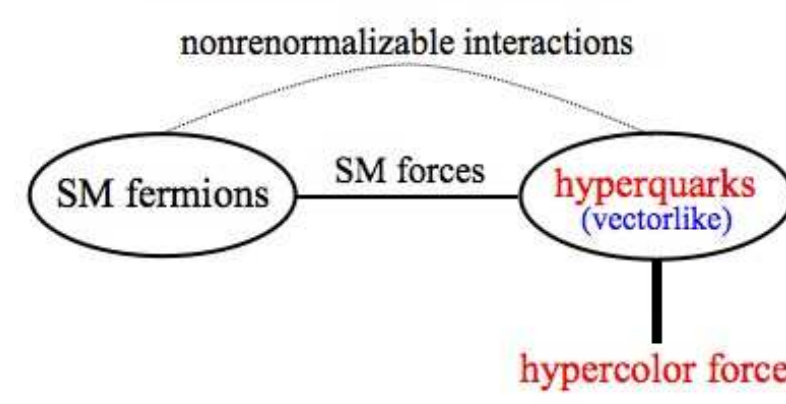

(QED-QCD system)

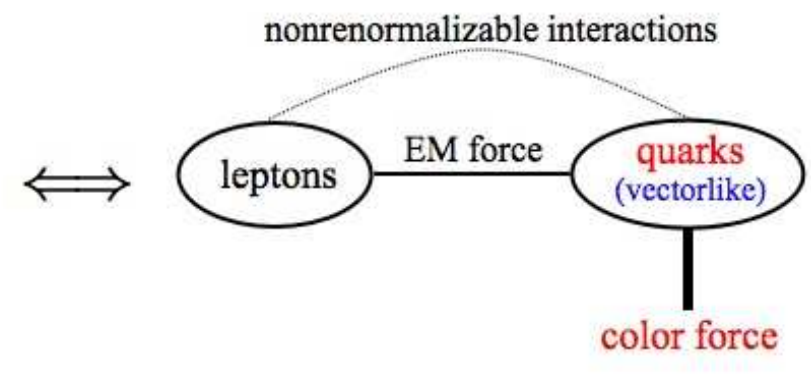

Figure 1: The analogy between the structure of Vectorlike Confinement and that of the QED-QCD system. Each oval represents a set of matter fields, while each line represents an interaction, thicker lines corresponding to stronger interactions. A line ending at an oval means that the oval feels the interaction represented by the line.

toriously difficult phenomenon to study quantitatively. Technicolor harnesses the power of strongly coupled gauge dynamics in a theoretically reliable way by imitating QCD itself and using strong interaction data as an "analog computer". We will follow essentially the same strategy, differing only in how the new physics couples to the SM gauge interactions.

This paper is aimed at highlighting some of the generic features of the rich phenomenology in the Vectorlike Confinement scenario as well as demonstrating consistency with all present experimental data, both in the form of exclusions from direct searches as well as the non-observation of any virtual effects. Further details of the phenomenology as well as an outlook for experimental searches will be presented in future work [16].

In section 2 we will quantitatively describe the short distance physics in the Vectorlike Confinement scenario. Next, we will set up an effective Lagrangian in section 3 to describe the physics relevant for the LHC. Readers who are more interested in the collider phenomenology than the theoretical details can skip section 2 and most of section 3 and jump directly to subsection 3.6 where we summarize the physics processes that are most relevant for the LHC. We go on to discuss several representative models of Vectorlike Confinement and their most salient features in section 4. Our conclusions will be summarized in section 5 .

\section{The Microscopic Structure of Vectorlike Confinement}

The fundamental Lagrangian of Vectorlike Confinement can be best described by drawing an analogy with the QED-QCD system (see Fig. 10). In the QED-QCD system, there are two types of matter, leptons and vectorlike quarks. There are three kinds of interactions, color force (strong), electromagnetic force (medium strength), and nonrenormalizable interactions (weak) such as 4-

fermion operators with two leptons and two quarks. The nonrenormalizable interactions are quite feeble, and their major role in phenomenology is to break otherwise conserved quantum numbers 
(such as "up number" and "down number") and let otherwise stable particles (such as $\pi^{ \pm}$) decay. If we ignore the nonrenormalizable interactions, the electromagnetic gauge interaction is the only interaction connecting quarks to leptons.

Similarly, in Vectorlike Confinement, we have two sets of matter, SM fermions and vectorlike "hyperquarks". Hyperquarks feel three types of interactions, a new confining gauge interaction "hypercolor" (strong), SM gauge interactions (medium strength), and nonrenormalizable interactions (weak). The nonrenormalizable interactions have very small effects, and their major phenomenological impact is to break otherwise exact symmetries and let otherwise stable particles decay. If we ignore the nonrenormalizable interactions, hyperquarks can interact with SM fermions only via SM gauge interactions 5

The vectorlike nature of hyperquarks is crucial. Just like color, hypercolor is assumed to confine and spontaneously break the axial flavor symmetry of hyperquarks while preserving the vector flavor symmetry. Since hyperquarks are vectorlike under SM gauge interactions, this "hyper-chiral symmetry breaking" does not break any of the SM gauge symmetries. This (and the absence of renormalizable Higgs-hyperquark couplings) guarantee that the hypercolor dynamics have negligible impact on electroweak precision observables [17, 18]. More systematically, note that, upon integrating out the hyper sector, the nonrenormalizable operators that parameterize corrections to precision electroweak observables (e.g. $\left(H^{\dagger} W_{\mu \nu} H\right) B^{\mu \nu}$ for the $S$ parameter) all involve $H$. But since hyperquarks interact with the SM only via gauge interactions and have no direct couplings to $H$, one sees that any diagram with hyperquarks that contributes to those operators must involve additional two loops beyond existing SM contributions. Note that hyperquark loops do contribute to electroweak-preserving vacuum polarizations such as $W^{\mu \nu} W_{\mu \nu}$ and $B^{\mu \nu} B_{\mu \nu}$, but their effects on precision electroweak observables cancel out once expressed in terms of the standard input parameters such as the fine structure constant, $G_{F}$ and $m_{Z}$, as is clear from the Peskin-Takeuchi analysis [18].

Another crucial feature depicted by Fig. 1 is that, in the limit of neglecting nonrenormalizable interactions, SM gauge interactions are the only interactions connecting hyperquarks to SM fermions. Then, in this limit, flavor violations beyond the SM are completely negligible, since new flavor-violating diagrams involving hyperquark loops are necessarily suppressed by additional two loop factors $\left(\sim 1 /\left(16 \pi^{2}\right)^{2}\right)$ compared to the existing flavor-violating diagrams in the SM. Nonrenormalizable operators, especially 4-fermion operators with two or four SM fermions, do in general induce new flavor violations, but they can be suppressed by simply taking the scale of these operators to be high.

Below, we will describe the theory of the Vectorlike Confinement scenario and analyze its dynamics by beginning with the strongest interaction, hypercolor, and then turning on weaker interactions, one by one, as perturbations.

\footnotetext{
${ }^{5}$ Ensuring this property requires in particular that the quantum numbers of hyperquarks be such that no Yukawa couplings with the Higgs are allowed for hyperquarks.
} 


\subsection{Hypercolor and Hyperflavor}

Let us begin with the strongest interaction, i.e. hypercolor (HC), and ignore all SM interactions and nonrenormalizable interactions. The Lagrangian in this limit reads simply

$$
\mathcal{L}=\mathcal{L}_{\mathrm{SM}}-\frac{1}{4} H_{\mu \nu}^{A} H^{A \mu \nu}+\sum_{i=1}^{F} \bar{\psi}_{i}\left(i \not D-m_{i}\right) \psi_{i},
$$

where $H_{\mu \nu}^{A}$ is the field strength for the $\mathrm{SU}(N)$ hypercolor gauge field $H_{\mu}^{A}\left(A=1, \cdots, N^{2}-1\right)$, and

$$
D_{\mu}=\partial_{\mu}+i g_{\mathrm{HC}} H_{\mu}^{A} t^{A}
$$

where $g_{\mathrm{HC}}$ is the hypercolor gauge coupling and $t^{A}$ is a generator of $\mathrm{SU}(N)$. There are $F$ Dirac fermions $\psi_{i}(i=1, \cdots, F)$, or "hyperquarks", all of which are assumed to be in the fundamental representation of $\mathrm{SU}(N)_{\mathrm{HC}} 6$ We refer to the variety of $\psi_{i}$ distinguished by $i$ as "hyperflavor", analogous to flavor in QCD. If we neglect hyperquark masses $m_{i}$, the theory has a global symmetry ("hyperflavor symmetry")

$$
\mathrm{SU}(F)_{\mathrm{L}} \otimes \mathrm{SU}(F)_{\mathrm{R}} \otimes \mathrm{U}(1)_{\mathrm{HB}}
$$

under which $\psi_{\mathrm{L}, \mathrm{R}} \equiv \frac{1 \mp \gamma_{5}}{2} \psi\left(\right.$ where $\left.\psi \equiv\left(\psi_{1}, \cdots, \psi_{F}\right)\right)$ transform as

$$
\begin{aligned}
\psi_{\mathrm{L}} & \longrightarrow \exp \left[i \alpha_{\mathrm{L}}^{a} T^{a}\right] \psi_{\mathrm{L}}, \\
\psi_{\mathrm{R}} & \longrightarrow \exp \left[i \alpha_{\mathrm{R}}^{a} T^{a}\right] \psi_{\mathrm{R}}, \\
\psi & \longrightarrow \exp \left[i \alpha_{\mathrm{HB}} / N\right] \psi,
\end{aligned}
$$

where $T^{a}\left(a=1, \cdots, F^{2}-1\right)$ are the generators of $\mathrm{SU}(F)$. U(1) $\mathrm{HB}$, or "hyperbaryon number", is analogous to the baryon number in QCD.

Like QCD, we assume that hypercolor confines and triggers spontaneous breaking of the hyperflavor symmetry (2.3) as:

$$
\mathrm{SU}(F)_{\mathrm{L}} \otimes \mathrm{SU}(F)_{\mathrm{R}} \otimes \mathrm{U}(1)_{\mathrm{HB}} \quad \longrightarrow \quad \mathrm{SU}(F)_{\mathrm{V}} \otimes \mathrm{U}(1)_{\mathrm{HB}}
$$

where $\mathrm{SU}(F)_{\mathrm{V}}$ is the subgroup satisfying $\alpha_{\mathrm{L}}=\alpha_{\mathrm{R}}$ in (2.4). Nonzero hyperquark masses $m_{i}$ do not significantly impact this symmetry breaking pattern as long as they are much smaller than the hypercolor confinement scale $\Lambda_{\mathrm{HC}}$. (Therefore, we define $F$ to be the number of light (« $\Lambda_{\mathrm{HC}}$ ) hyperquarks.) Note that the value of $F$ must be consistent (i.e. sufficiently small) with the occurrence of hypercolor confinement and "hyper-chiral symmetry breaking" (2.5).

Analogously to QCD, hyper-chiral symmetry breaking yields $F^{2}-1$ (Pseudo)-Nambu-Goldstone bosons transforming altogether as an adjoint multiplet of $\mathrm{SU}(F)_{\mathrm{V}}$ (with small masses induced by $m_{i}$ ). We refer to them as "hyperpions". Being much lighter than all other bound states of hyperquarks, hyperpions play an important role in collider phenomenology.

\footnotetext{
${ }^{6}$ If we choose a non-complex representation of $\mathrm{SU}(N)_{\mathrm{HC}}$ for hyperquarks, or if we choose a non-complex group like $\mathrm{SO}(N)$ or $\operatorname{Sp}(N)$ for hypercolor, then the hyperflavor breaking pattern 2.5 would be changed and the analogy with the QED-QCD system must be used with care. We will not pursue such alternatives in this paper.
} 


\subsection{SM Gauge Interactions and the Concept of "Species"}

Let us now turn on SM gauge interactions. The fundamental Lagrangian now reads

$$
\mathcal{L}=\mathcal{L}_{\mathrm{SM}}-\frac{1}{4} H_{\mu \nu}^{A} H^{A \mu \nu}+\sum_{I=1}^{S} \bar{\Psi}_{I}\left(i \not D-m_{I}\right) \Psi_{I},
$$

where

$$
D_{\mu} \Psi_{I}=\partial_{\mu} \Psi_{I}+i g_{\mathrm{HC}} H_{\mu}^{A} t^{A} \Psi_{I}+i \sum_{G} g_{G} A_{\mu}^{(G) \alpha} T_{\Psi_{I}}^{(G) \alpha} \Psi_{I}
$$

where $T_{\Psi_{I}}^{(G)}$ are the generators of the $\mathrm{SM}$ gauge group $G=\mathrm{SU}(3)_{\mathrm{C}}, \mathrm{SU}(2)_{\mathrm{W}}, \mathrm{U}(1)_{\mathrm{Y}}$ for $\Psi_{I}$. Note that $\Psi_{I}$ is different from $\psi_{i}$ in (2.1); the $F$ flavors, $\psi_{1}, \psi_{2}, \cdots, \psi_{F}$, have reorganized themselves into $S$ multiplets, $\Psi_{1}, \Psi_{2}, \cdots, \Psi_{S}$, of the SM gauge group. We refer to the variety of $\Psi_{I}$ distinguished by $I$ as "species". For example, one could imagine a 5 -flavor $(F=5)$ model where the 5 flavors reorganize themselves into two species $(S=2)$, say, a triplet of color and a doublet of weak interaction 7 Now that the $\Psi$ are charged under the SM, one must make sure that their impact on the running of SM gauge couplings is small enough to avoid Landau poles near the TeV scale.

By our assumption the $\Psi$ are all vectorlike under the SM gauge group, that is, $T_{\Psi_{I}}^{(G)}$ all belong to $\mathrm{SU}(F)_{\mathrm{V}}$. Therefore, $\mathrm{SM}$ gauge interactions explicitly break the axial part of $\mathrm{SU}(F)_{\mathrm{L}} \otimes \mathrm{SU}(F)_{\mathrm{R}}$, so hyperpions that carry SM charges are not exact Nambu-Goldstone bosons even if $m_{I}=0$. Consequently, non-singlet hyperpions acquire mass contributions depending on their SM gauge quantum numbers. This is analogous to the difference $m_{\pi^{ \pm}}^{2}-m_{\pi^{0}}^{2}$ in the QED-QCD system, which is induced by QED. Note that the radiative contributions from the SM gauge sector can be the dominant source of mass for hyperpions, whereas in QCD the up and down quark masses are large enough to be the dominant source of pion masses.

\subsubsection{Mixing of Vector Mesons with SM Gauge Bosons}

The perturbation added in going from (2.1) to (2.6) is

$$
\Delta \mathcal{L}=-\sum_{G} g_{G} A_{\mu}^{(G) \alpha} J_{G}^{\alpha \mu}
$$

where $G=\mathrm{SU}(3)_{\mathrm{C}}, \mathrm{SU}(2)_{\mathrm{W}}, \mathrm{U}(1)_{\mathrm{Y}}$, and

$$
J_{G}^{\alpha \mu}=\sum_{I=1}^{S} \bar{\Psi}_{I} \gamma^{\mu} T_{\Psi_{I}}^{(G) \alpha} \Psi_{I} .
$$

Now, analogously to $\rho$ mesons in QCD, the Noether currents corresponding to $\mathrm{SU}(F)_{\mathrm{V}} \otimes \mathrm{U}(1)_{\mathrm{HB}}$ can create parity-odd spin-1 bound states of a hyperquark and an anti-hyperquark, or "hyper- $\rho$ mesons" for short, with masses $m_{\tilde{\rho}} \sim \Lambda_{\mathrm{HC}}$. Since $J_{G}^{\alpha \mu}$ are a subset of these vector currents, the interactions (2.8) lead to mixings between SM gauge bosons and the hyper- $\rho$ mesons interpolated by $J_{G}^{\mu}$. In the QED-QCD system, such a mixing occurs between the $\gamma, \rho^{0}$ and $\omega$.

\footnotetext{
${ }^{7}$ The distinction between species and flavors is obscured in the QED-QCD analogy. In this analogy, $G=\mathrm{U}(1)_{\mathrm{EM}}$ and there are three species $u, d, s$ with $T_{u}=2 / 3, T_{d}=-1 / 3, T_{s}=-1 / 3$. But since $\mathrm{U}(1)$ has only one-dimensional representations, species coincide with flavors.
} 
The hyper- $\rho$ mesons corresponding to $J_{G}^{\alpha \mu}$ have a special status, as they can be singly produced via mixing with an $s$-channel SM gauge boson, while the rest of hyper- $\rho$ 's and all other massive hyper-hadrons must be pair-produced. Therefore, together with the hyperpions, which are light, these hyper- $\rho$ 's dominate the collider phenomenology.

Let us revisit the corrections to precision electroweak observables and see why they are kept small in Vectorlike Confinement, this time from the "hadronic" viewpoint of $\tilde{\rho}$-SM-gauge-boson mixing. New physics contributions to the "oblique" parameters, i.e. vacuum polarizations of electroweak gauge bosons, are dominated by the mixing of electroweak gauge bosons to the $\tilde{\rho}$ and back again. Since the $\tilde{\rho}$ are much heavier than the $Z$ mass, the oblique corrections are well parameterized by the Peskin-Takeuchi $S, T$ and $U$ parameters [18. Since $T$ and $U$ measure custodial isospin violation and the hyper sector explicitly preserves this symmetry, we need only be concerned with the $S$ parameter. $S$ is essentially the strength of kinetic mixing $W_{\mu \nu}^{3} B^{\mu \nu}$ after electroweak symmetry breaking, which feeds into precision observables such as the $W$ mass measurement. However in our case, the new physics does not break electroweak symmetry itself, and neither does it couple to the Higgs, so mixing with the $\tilde{\rho}$ only induces the electroweak-preserving polarization effects, $W_{\mu \nu} W^{\mu \nu}$ and $B_{\mu \nu} B^{\mu \nu}$, which however do not feed into precision electroweak observables as we already discussed at the beginning of Sec. 2 .

\subsubsection{Accidental "Species Symmetries" and Long-lived Hyperpions}

In the renormalizable Lagrangian (2.6), the $\mathrm{SM}$ gauge interactions explicitly break $\mathrm{SU}(F)_{\mathrm{V}} \otimes \mathrm{U}(1)_{\mathrm{HB}}$ flavor symmetry, but the subgroup $\mathrm{U}(1)_{\Psi_{1}} \otimes \mathrm{U}(1)_{\Psi_{2}} \otimes \cdots \otimes \mathrm{U}(1)_{\Psi_{S}}$ are still preserved. Therefore, " $\Psi_{1}$ number", " $\Psi_{2}$ number", ..., " $\Psi_{S}$ number", or collectively "species numbers", are all conserved 8 A special case is the hyperbaryon number, which is just the average of all species numbers.

Species number conservation has profound phenomenological consequences. A hyperpion with the quantum numbers of $\bar{\Psi}_{I} \Psi_{J}(I \neq J)$ cannot decay to SM particles due to its nonzero species numbers. In the QED-QCD analogy of Fig. 1, the $\pi^{ \pm}$would be exactly stable if the nonrenormalizable interactions were ignored, due to the conservation of "up number" and "down number". On the other hand, a hyperpion of the $\bar{\Psi}_{I} \Psi_{I}$ type carries no species numbers and will promptly decay to a pair of SM gauge bosons, just like $\pi^{0} \rightarrow \gamma \gamma$ in the QED-QCD system.

Of course, species number conservation is an accidental feature of the renormalizable Lagrangian (2.6), so these stable particles may eventually decay via a nonrenormalizable interaction, just like the $\pi^{ \pm}$eventually decays to leptons via a 4 -fermion operator. This is the topic of the following subsection.

\footnotetext{
${ }^{8} \mathrm{~A}$ species symmetry could in principle be larger than $\mathrm{U}(1)$ if there are more than one species with identical SM gauge quantum numbers and masses. For example, if $\Psi_{1}$ and $\Psi_{2}$ have the same gauge quantum numbers and masses, they would have a $\mathrm{U}(2)$ species symmetry, instead of $\mathrm{U}(1)_{\Psi_{1}} \otimes \mathrm{U}(1)_{\Psi_{2}}$. However, unlike $\mathrm{U}(1)$ species symmetries, which are automatic in the renormalizable Lagrangian (2.6), a non-Abelian species symmetry requires tuning of masses and therefore does not enjoy the privilege of accidental symmetry. While one could remove the tuning by gauging the species symmetry, which may give rise to interesting phenomenology, we will not pursue this possibility further in this paper.
} 


\subsection{Nonrenormalizable Interactions}

We finally come to the last ingredient depicted in Fig. 1, namely nonrenormalizable operators connecting the SM and the hyper sector. Nonrenormalizable interactions are of course expected to exist at some level, and while most of them do not qualitatively affect the physics, some of them do as they induce processes forbidden in the renormalizable Lagrangian (2.6), such as those which violate species numbers.

\subsubsection{Mixed SM-hyper 4-fermion Interactions and Hyperpion Decays}

In the QED-QCD system, the 4-fermion operators $\left(\bar{d} \gamma_{\mu} \gamma_{5} u\right)\left(\bar{\nu}_{\ell} \bar{\sigma}^{\mu} \ell\right)$ with $\ell=e, \mu$ are the leading interactions which break the up and down numbers and allow a $\pi^{+}$to decay. Similarly, the lifetimes of hyperpions with nonzero species numbers are controlled by the 4-fermion interactions involving two hyperquarks and two SM fermions which break species symmetries 9

Suppose the $\tilde{\pi}$ with the quantum numbers of $\bar{\Psi}_{I} \Psi_{J}$ decays to a pair of SM fermions, either $\bar{f}_{i} f_{j}^{\prime}$ or $f_{i} f_{j}^{\prime}\left(f, f^{\prime}=q, u^{c}, d^{c}, \ell, e^{c}\right.$ and $\left.i, j=1,2,3\right)$, whichever the quantum numbers match. The 4-fermion operator mediating the $\tilde{\pi}_{\bar{I} J} \rightarrow \bar{f}_{i} f_{j}^{\prime}$ decay takes the "current-current" form:

$$
\frac{C_{\tilde{\pi}_{\bar{J} I} \bar{f}_{i} f_{j}}}{M^{2}}\left(\bar{\Psi}_{J} \gamma^{\mu} \gamma_{5} \Psi_{I}\right)\left(\bar{f}_{i} \bar{\sigma}_{\mu} f_{j}^{\prime}\right)
$$

while the one for $\tilde{\pi}_{\bar{I} J} \rightarrow f_{i} f_{j}^{\prime}$ takes the "scalar-scalar" form:

$$
\frac{C_{\tilde{\pi}_{\bar{J}} f_{i} f_{j}}}{M^{2}}\left(\bar{\Psi}_{J} \gamma_{5} \Psi_{I}\right)\left(f_{i} f_{j}^{\prime}\right) .
$$

Recall that in the QED-QCD analogy, the $\pi^{ \pm}$decays to leptons are induced by the current-current 4 -fermion operators 10

The scale suppressing these mixed SM-hyper 4-fermion interactions cannot be too low. Using any one of these vertices twice and closing the hyperquarks in a loop will induce a purely-SM 4 -fermion operator. Unless we make some extra assumptions governing the flavor structures of $C_{\tilde{\pi}_{\bar{J} I} \bar{f}_{i} f_{j}}$ and $C_{\tilde{\pi}_{\bar{J} I} f_{i} f_{j}}$, the induced purely-SM 4-fermion operators generically lead to flavor-changing neutral currents (FCNCs). The absence of FCNCs then requires the coefficients of these operators be $\lesssim\left(\mathcal{O}\left(10^{5}\right) \mathrm{TeV}\right)^{-2}$ [19]. For $C_{\tilde{\pi}_{\bar{J} I} \bar{f}_{i} f_{j}} \sim C_{\tilde{\pi}_{\bar{J} I} f_{i} f_{j}} \sim \mathcal{O}(1)$, this translates to the bound $M \gtrsim$ $\mathcal{O}\left(10^{5} / 4 \pi\right) \mathrm{TeV} \sim \mathcal{O}\left(10^{4}\right) \mathrm{TeV}$. If we do assume an extra flavor symmetry, such as $C_{\tilde{\pi}_{\bar{J}} \bar{f}_{i} f_{j}} \propto$ $C_{\tilde{\pi}_{\bar{J} I} f_{i} f_{j}} \propto \delta_{i j}$, then $M$ needs to be only high enough for the effective field theory description of Fig. 1 to make sense.

\subsubsection{Hyperbaryon Decay}

Hyperbaryon number $\mathrm{U}(1)_{\mathrm{HB}}$ is also an accidental symmetry of the renormalizable lagrangian (2.6). While all hypermesons are neutral under $\mathrm{U}(1)_{\mathrm{HB}}$, "hyperbaryon" operators, i.e. the products of $N$

\footnotetext{
${ }^{\mathbf{9}}$ It is in principle possible that a particular model may not allow any such 4-fermion operators that are gauge invariant and one would have to consider even higher dimensional operators. For definiteness, we stick to the 4-fermion case in this paper.

${ }^{10}$ Of course, in the QED-QCD analogy the physics underlying the 4-fermion weak interactions ultimately is related to the origin of quark masses. In our case, we will stay agnostic as to whether our 4-fermion operators are deeply connected to the origin of hyperfermion masses or not. For the phenomenology we will pursue, what matters is that they in general can break accidentally conserved global quantum numbers of the renormalizable dynamics.
} 
hyperquark fields, carry a unit hyperbaryon number. Since the properties of hyperbaryons strongly depend on the details such as $N$ and $F$, we will discuss their decays in each specific model below.

\section{Phenomenological Lagrangian for the LHC}

In this section, we write down a crude Lagrangian describing the interactions of hyperpions $(\tilde{\pi})$ and hyper- $\rho$ mesons $(\tilde{\rho})$ with the SM particles and among themselves. This description is good enough for a rough understanding of the LHC phenomenology of the theory introduced in Sec. 2. It is not meant to be a systematic effective Lagrangian controlled by small expansion parameters. There is some rationale for including the $\tilde{\rho}$ but not other massive hyper-hadrons, even though their masses are similar to those of the $\tilde{\rho}$. As we already pointed out in Sec. 2.2.1, a $\tilde{\rho}$ can be singly produced in the collision of SM particles, while all other massive hyperhadrons must be pair-produced, so their mass scale is effectively $\approx 2 m_{\tilde{\rho}}$ as far as collider phenomenology is concerned. Therefore, at hadron colliders, the signatures of the hyper sector are dominated by the resonant production of a $\tilde{\rho}$ and its subsequent decay to a $\tilde{\pi}$ pair, as well as the "Drell-Yan" production of a $\tilde{\pi}$ pair from SM gauge interactions. In this spirit, there is no need to include the $\tilde{\rho}$ which do not mix with SM gauge bosons as they can only be pair-produced, so our phenomenological Lagrangian captures all interactions that are relevant for the production and decay of the hypercolor bound states that can be produced at energies below $\Lambda_{H C}$.

In the following, we will go through the various features of the following phenomenological Lagrangian:

$$
\begin{aligned}
\mathcal{L}_{\text {eff }}= & \mathcal{L}_{\mathrm{SM}} \\
& +\sum_{G}\left[-\frac{1}{4} \tilde{\rho}_{\mu \nu}^{(G) \alpha} \tilde{\rho}^{(G) \alpha \mu \nu}+\frac{m_{\tilde{\rho}}^{2}}{2} \tilde{\rho}_{\mu}^{(G) \alpha} \tilde{\rho}^{(G) \alpha \mu}-\frac{\varepsilon_{G}}{2} \tilde{\rho}_{\mu \nu}^{(G) \alpha} F^{(G) \alpha \mu \nu]}\right. \\
& +\sum_{P=1}^{S_{\tilde{\pi}}} \frac{1}{2^{c_{P}}}\left[\left(D_{\mu} \tilde{\pi}_{P}\right)^{\dagger}\left(D^{\mu} \tilde{\pi}_{P}\right)-m_{\tilde{\pi}_{P}}^{2} \tilde{\pi}_{P}^{\dagger} \tilde{\pi}_{P}\right] \\
& -g_{\tilde{\rho} \tilde{\pi} \tilde{\pi} f}^{a b c} \tilde{\rho}_{\mu}^{a} \tilde{\pi}^{b} D^{\mu} \tilde{\pi}^{c} \\
& -\frac{N \epsilon^{\mu \nu \rho \sigma}}{16 \pi^{2} f_{\tilde{\pi}}} \sum_{G, G^{\prime}} g_{G} g_{G^{\prime}} \operatorname{tr}\left[\tilde{\pi}^{a} T^{a} F_{\mu \nu}^{(G)} F_{\rho \sigma}^{\left(G^{\prime}\right)}\right] \\
& +\mathcal{L}_{\tilde{\pi} f \bar{f}}
\end{aligned}
$$

\subsection{Production and Decay of $\tilde{\rho}$}

The first and second terms in (3.1a) are respectively the kinetic and mass terms of $\tilde{\rho}_{\mu}^{(G) \alpha}$, where $G=\mathrm{SU}(3)_{\mathrm{C}}, \mathrm{SU}(2)_{\mathrm{W}}, \mathrm{U}(1)_{\mathrm{Y}}$ and $\alpha$ labels the generators of each $G$. The third term describes the mixing of $\tilde{\rho}_{\mu}^{(G)}$ with the SM gauge boson $A_{\mu}^{(G)}$ (whose field strength is $F_{\mu \nu}^{(G)}$ ). The precise value of the mixing parameter $\varepsilon_{G}$ depends on the details of the hyper sector, but a rough estimate based on standard large- $N$ counting is:

$$
\varepsilon_{G} \approx \frac{\sqrt{N}}{4 \pi} g_{G}
$$

For practical calculations it is more convenient to eliminate the mixing term in (3.1a) and work in the "diagonal" basis of $A_{\mu}^{(G)}$ and $\tilde{\rho}_{\mu}^{(G)}$. This can be done (up to $\mathcal{O}\left(\varepsilon_{G}^{2}\right)$ corrections) by shifting $A_{\mu}^{(G)}$ 
as

$$
A_{\mu}^{(G)} \longrightarrow A_{\mu}^{(G)}-\varepsilon_{G} \tilde{\rho}_{\mu}^{(G)}
$$

These shifts induce couplings of the $\tilde{\rho}_{\mu}^{(G)}$ to SM fermions $f=q, u^{c}, d^{c}, \ell, e^{c}$ :

$$
\mathcal{L}_{\tilde{\rho} f \bar{f}}=\sum_{f} \sum_{G} \varepsilon_{G} g_{G} \bar{f} \bar{\sigma}^{\mu} \tilde{\rho}_{\mu}^{(G)} f .
$$

We briefly remark here that while (3.4) allows resonant production of a $\tilde{\rho}$ from a $q-\bar{q}$ initial state, gauge invariance does not allow for a colored $\tilde{\rho}$ to be resonantly produced from a gluon-gluon initial state via renormalizable couplings. There are however operators of higher mass dimension that can induce such a coupling, which are not included in (3.1). In [5], such an operator was studied in order to estimate any additional contribution to the production cross section. The impact on the phenomenology was found to be a minor one.

Once the $\tilde{\rho}$ are resonantly produced, they will decay dominantly to a pair of $\tilde{\pi}$ as described by the interaction (3.1c), where $a, b, c=1, \cdots, F^{2}-1$ and $f^{a b c}$ is the structure constant of $\mathrm{SU}(F)_{\mathrm{V}}$. The $\tilde{\rho} \rightarrow \tilde{\pi} \tilde{\pi}$ rate (summed over all $\tilde{\pi}$ ) can readily be computed as

$$
\Gamma_{\tilde{\rho} \rightarrow \tilde{\pi} \tilde{\pi}}=\frac{m_{\tilde{\rho}} g_{\tilde{\rho} \tilde{\pi} \tilde{\pi}}^{2} F}{96 \pi}\left(1-\frac{4 m_{\tilde{\pi}}^{2}}{m_{\tilde{\rho}}^{2}}\right)^{3 / 2},
$$

where we have neglected differences among $m_{\tilde{\pi}}$ 's for simplicity. The coupling strength $g_{\tilde{\rho} \tilde{\pi} \tilde{\pi}}$ can be estimated as

$$
g_{\tilde{\rho} \tilde{\pi} \tilde{\pi}} \approx \frac{4 \pi}{\sqrt{N}} .
$$

Using this estimate with $N=3$, (3.5) agrees well with the observed $\rho \rightarrow \pi \pi$ rate in the QED-QCD system.

The $\tilde{\rho}$ can also decay to SM fermions via (3.4). Neglecting the $f$ mass, the partial width per Weyl fermion $f$ is given by

$$
\Gamma_{\tilde{\rho}^{(G)} \rightarrow f \bar{f}}=\frac{g_{G}^{2} \varepsilon_{G}^{2}}{24 \pi} D_{f} m_{\tilde{\rho}},
$$

where $D_{f}=1 / 2$ for $G=\mathrm{SU}(3)_{\mathrm{C}}, \mathrm{SU}(2)_{\mathrm{W}}$ while $D_{f}=Y_{f}^{2}$ for $G=\mathrm{U}(1)_{\mathrm{Y}}$. (Estimating $\varepsilon_{G}$ from (3.2) with $N=3$ and $G=\mathrm{U}(1)_{\mathrm{EM}}$, the above formula agrees well with the partial width of $\rho^{0} \rightarrow e^{+} e^{-}$

in the QED-QCD system.) Note that unless $N$ is very large, the decay into SM fermions is very suppressed and the $\tilde{\rho}$ width is well approximated by (3.5).

\subsection{Sources of Hyperpion Masses}

Next, (3.1b) describes the kinetic and mass terms of $\tilde{\pi}_{P}$, where $P=1, \cdots, S_{\tilde{\pi}}$ labels different species of $\tilde{\pi}$. (When the $F \psi$ 's reorganize themselves into the $S \Psi$ 's, the $F^{2}-1 \tilde{\pi}^{\prime}$ 's accordingly reorganize themselves into $S_{\tilde{\pi}}$ multiplets of the SM gauge group.) The number $c_{P}$ is 0 for a complex $\tilde{\pi}_{P}$ and 1 for a real $\tilde{\pi}_{P}$. The $\mathrm{SM}$ gauge interactions of $\tilde{\pi}_{P}$ are encoded in

$$
D_{\mu} \tilde{\pi}_{P}=\partial_{\mu} \tilde{\pi}_{P}+i \sum_{G} g_{G} A_{\mu}^{(G) \alpha} T_{\tilde{\pi}_{P}}^{(G) \alpha} \tilde{\pi}_{P}
$$


where $T_{\tilde{\pi}_{P}}^{(G)}$ are the generators of the SM gauge group $G$ for $\tilde{\pi}_{P}$. There are three sources of $\tilde{\pi}$ masses. First, as we mentioned in Sec. 2.2, the $\tilde{\pi}$ are not exact Nambu-Goldstone bosons even when all hyperquark masses $m_{I}$ are zero in (2.6), because SM gauge interactions explicitly break the axial part of $\mathrm{SU}(F)_{\mathrm{L}} \otimes \mathrm{SU}(F)_{\mathrm{R}}$. These contributions are given by

$$
\left.m_{\tilde{\pi}_{P}}^{2}\right|_{\text {gauge }}=\frac{3 c^{2} m_{\tilde{\rho}}^{2}}{16 \pi^{2}} \sum_{G} g_{G}^{2} C_{2}^{(G)}\left(\tilde{\pi}_{P}\right),
$$

where $C_{2}^{(G)}\left(\tilde{\pi}_{P}\right)$ is the quadratic Casimir of the SM gauge group $G$ for $\tilde{\pi}_{P}$. The constant $c$ is an $\mathcal{O}(1)$ number whose precise value depends on the details of the hyper sector. (For $N=F=3$, we obtain $c \simeq 1.10$ from $m_{\pi^{ \pm}}^{2}-m_{\pi^{0}}^{2}$ in the QED-QCD system.) The second source of $m_{\tilde{\pi}}$ is the nonzero (but $\ll m_{\tilde{\rho}}$ ) hyperquark masses, giving rise to

$$
\left.m_{\tilde{\pi}_{P}}^{2}\right|_{\text {h.q. mass }} \approx m_{\tilde{\rho}} m_{\mathrm{hq}},
$$

where we have taken $m_{I}=m_{\mathrm{hq}}$ for all $I$ for simplicity. The last possible source of $m_{\tilde{\pi}}$ is electroweak symmetry breaking (EWSB). If a $\tilde{\pi}_{P}$ is charged under $\mathrm{SU}(2)_{\mathrm{W}}, W$ and $Z$ loops after EWSB split the masses of different $\mathrm{SU}(2)_{\mathrm{W}}$ components of the $\tilde{\pi}_{P}$. The leading contributions are IR dominated and thus calculable [20]:

$$
\Delta m_{\tilde{\pi}} \simeq \alpha_{2} M_{Z} Q_{\mathrm{diff}}\left[Q_{\mathrm{avg}} \sin ^{2} \theta_{W}-\left(Q_{\mathrm{avg}}-Y\right)\left(1-\cos \theta_{W}\right)\right]
$$

where $\Delta m_{\tilde{\pi}}$ is the mass splitting between any two components of the $\tilde{\pi}_{P}$, and $Q_{\text {diff }}$ and $Q_{\text {avg }}$ are respectively the difference and average of the electric charges of the two components, and $Y$ is the common hypercharge. These mass splittings are typically of $\mathcal{O}(100) \mathrm{MeV}$, but can significantly affect collider phenomenology because a heavier component can decay to a lighter component by emitting an off-shell $W$.

\subsection{Decays of Short-lived Hyperpions}

The interactions (3.1d) represent the chiral anomalies of the underlying $\mathrm{SU}(F)_{\mathrm{L}} \otimes \mathrm{SU}(F)_{\mathrm{R}}$ in the presence of SM gauge fields (hence the trace is taken over the $\mathrm{SU}(F)_{\mathrm{V}}$ space, on which the SM gauge groups act). In the language of Feynman diagrams, the triangle diagram exists only when the $\Psi$ loop can be closed, thus these vertices exist only for the $\tilde{\pi}$ made out of the same species of $\Psi$, i.e. precisely the $\tilde{\pi}$ that carry no net species number. Such $\tilde{\pi}$ will decay promptly to a pair of SM gauge bosons. In the QED-QCD analogy, this corresponds to the well known chiral anomaly responsible for $\pi^{0} \rightarrow \gamma \gamma$. The $\tilde{\pi}$ decay constant $f_{\tilde{\pi}}$ is normalized as

$$
\left\langle 0\left|\bar{\Psi} \gamma_{\mu} \gamma_{5} T^{a} \Psi(x)\right| \tilde{\pi}^{b}(p)\right\rangle \equiv-i f_{\tilde{\pi}} \delta^{a b} p_{\mu} e^{-i p \cdot x},
$$

where the $\mathrm{SU}(F)$ generators $T^{a}$ are normalized as $\operatorname{tr}\left[T^{a} T^{b}\right]=\delta^{a b} / 2$. (This is the same as the convention where $f_{\pi}=92 \mathrm{MeV}$ in the QED-QCD system.) A rough estimate for $f_{\tilde{\pi}}$ is

$$
f_{\tilde{\pi}} \approx \frac{m_{\tilde{\rho}} \sqrt{N}}{4 \pi} .
$$




\subsection{Decays of Long-lived Hyperpions}

Finally, $\mathcal{L}_{\tilde{\pi} f \bar{f}}$ in (3.1) describes the interactions which violate species symmetries, thereby allowing the otherwise stable $\tilde{\pi}$ to decay. In calculating the matrix element for $\tilde{\pi}_{\bar{I} J} \rightarrow \bar{f}_{i} f_{j}^{\prime}$, the currentcurrent operator (2.10) reduces to

$$
\approx \frac{C_{\tilde{\pi}_{\bar{I} J} \bar{f}_{i} f_{j}}}{M^{2}} f_{\tilde{\pi}} p_{\tilde{\pi}}^{\mu} \tilde{\pi}_{\bar{I} J}^{\dagger} \bar{f}_{i} \bar{\sigma}_{\mu} f_{j}^{\prime}
$$

by using the definition (3.12). On the other hand, for $\tilde{\pi}_{\bar{I} J} \rightarrow f_{i} f_{j}^{\prime}$, the scalar-scalar operator (2.11) reduces to

$$
\approx \frac{C_{\tilde{\pi}_{\bar{I} J} f_{i} f_{j}}}{M^{2}} f_{\tilde{\pi}} m_{\tilde{\rho}} \tilde{\pi}_{\bar{I} J}^{\dagger} f_{i} f_{j}^{\prime}
$$

Therefore, these decays can be described by including the following effective vertices in $\mathcal{L}_{\tilde{\pi} f \bar{f}}$ :

$$
\mathcal{L}_{\tilde{\pi} f \bar{f}}=\sum_{\tilde{\pi}, f_{i}, f_{j}^{\prime}} \frac{C_{\tilde{\pi} \bar{f}_{i} f_{j}^{\prime}} f_{\tilde{\pi}}}{M^{2}}\left(\partial_{\mu} \tilde{\pi}^{\dagger}\right) \bar{f}_{i} \bar{\sigma}^{\mu} f_{j}^{\prime}+\sum_{\tilde{\pi}, f_{i}, f_{j}^{\prime}} \frac{C_{\tilde{\pi} f_{i} f_{j}^{\prime}} f_{\tilde{\pi}} m_{\tilde{\rho}}}{M^{2}} \tilde{\pi}^{\dagger} f_{i} f_{j}^{\prime},
$$

where $f, f^{\prime}=q, u^{c}, d^{c}, \ell, e^{c}$ and $i, j=1,2,3$. The decay rate for $\tilde{\pi} \rightarrow \bar{f}_{i} f_{j}^{\prime}$ is then given (up to a group theory factor) by

$$
\begin{aligned}
\Gamma_{\tilde{\pi} \rightarrow \bar{f}_{i} f_{j}^{\prime}} & =\frac{C_{\tilde{\pi} \bar{f}_{i} f_{j}^{\prime}}^{2}}{16 \pi} \frac{m_{f_{i}}^{2}+m_{f_{j}^{\prime}}^{2}}{m_{\tilde{\pi}}^{2}} \frac{f_{\tilde{\pi}}^{2} m_{\tilde{\pi}}^{2}}{M^{4}} m_{\tilde{\pi}} \\
& \approx\left[10^{8} \mathrm{~m} \times \frac{1}{C_{\tilde{\pi} \bar{f}_{i} f_{j}^{\prime}}^{2} N} \frac{\mathrm{GeV}^{2}}{m_{f_{i}}^{2}+m_{f_{j}^{\prime}}^{2}} \frac{100 \mathrm{GeV}}{m_{\tilde{\pi}}}\left(\frac{\mathrm{TeV}}{m_{\tilde{\rho}}}\right)^{2}\left(\frac{M}{10^{4} \mathrm{TeV}}\right)^{4}\right]^{-1},
\end{aligned}
$$

where the estimate (3.13) for $f_{\tilde{\pi}}$ has been used, and we have assumed $m_{f_{i}}, m_{f_{j}^{\prime}} \ll m_{\tilde{\pi}}$. Note that the necessity for the chirality flip gives the suppression $m_{f_{i}}^{2}+m_{f_{j}^{\prime}}^{2} / m_{\tilde{\pi}}^{2}$, exactly analogous to the dominance of $\pi^{+} \rightarrow \mu^{+}+\nu_{\mu}$ over $\pi^{+} \rightarrow e^{+}+\nu_{e}$ in the QED-QCD system. On the other hand, the rate for $\tilde{\pi}_{\bar{I} J} \rightarrow f_{i} f_{j}^{\prime}$ is given (again up to a group theory factor) by

$$
\begin{aligned}
\Gamma_{\tilde{\pi} \rightarrow f_{i} f_{j}^{\prime}} & =\frac{C_{\tilde{\pi} f_{i} f_{j}^{\prime}}^{2} \frac{f_{\tilde{\pi}}^{2} m_{\tilde{\rho}}^{2}}{16 \pi} m_{\tilde{\pi}}}{M^{4}} \\
& \approx\left[100 \mathrm{~m} \times \frac{1}{C_{\tilde{\pi} f_{i} f_{j}^{\prime}}^{2} N} \frac{100 \mathrm{GeV}}{m_{\tilde{\pi}}}\left(\frac{\mathrm{TeV}}{m_{\tilde{\rho}}}\right)^{4}\left(\frac{M}{10^{4} \mathrm{TeV}}\right)^{4}\right]^{-1},
\end{aligned}
$$

where again we have assumed $m_{f_{i}}, m_{f_{j}^{\prime}} \ll m_{\tilde{\pi}}$.

To summarize, we can safely conclude that the $\tilde{\pi}$ carrying net species number are stable on collider time scales. It is possible for such $\tilde{\pi}$ to decay within the detector to SM fermion pairs, but in that case avoiding constraints from flavor violation requires that a nontrivial flavor structure be imposed on the coefficients $C_{\tilde{\pi} \bar{f}_{i} f_{j}^{\prime}}$ and $C_{\tilde{\pi} f_{i} f_{j}^{\prime}}$. Since the decay rates depend on the scale $M$ with a high power, displaced vertices would be visible in a narrow range for $M$ only. 


\subsection{Typical Constraints}

Let us now discuss a few generic constraints on the Vectorlike Confinement scenario. First, integrating out the $\tilde{\rho}$ which mix with SM gauge bosons yields flavor-universal 4-fermion operators, which are constrained by "fermion compositeness" searches. From the couplings (3.4), the coefficients of the 4-fermion operators become (up to $\mathcal{O}(1)$ SM group theory factors)

$$
\frac{\varepsilon_{G}^{2} g_{G}^{2}}{m_{\tilde{\rho}}^{2}} \approx \frac{N \alpha_{G}^{2}}{m_{\tilde{\rho}}^{2}} \equiv \frac{2 \pi}{\Lambda_{\mathrm{comp}}^{2}},
$$

where we have used (3.2). $\Lambda_{\text {comp }}$ can then be directly compared with the bounds listed in PDG [21]. The most severely constrained operators are of the form eeqq, which arise if there is a $\tilde{\rho}$ that mixes with the $Z$, and for $N=3$ the bounds require us to take $m_{\tilde{\rho}}>500 \mathrm{GeV}$.

When a model contains $\tilde{\pi}$ 's that decay to $W$ 's, $Z$ 's or $\gamma$ 's, it can be constrained by the searches at the Tevatron for such states [22]. It turns out that for the masses above 250-300 GeV the Tevatron does not have sensitivity to discover such $\tilde{\pi}$ because they are only electroweak charged and must be pair-produced, requiring higher center-of-mass energies. Note that since these decays are prompt, the Tevatron searches for long-lived particles decaying to $\gamma$ 's [23] or Z's [24] do not apply.

For long-lived $\tilde{\pi}$ that are charged or colored, we will always take the scale of the operators in (3.16) such that the lifetimes are small compared to cosmological timescales. This ensures that there is no conflict with the strong constraints from ocean-bottom searches [25] 29]. Collider constraints for the production of collider-stable charged or colored particles will be mentioned in the discussion of representative models in the next section, the result being that the mass scales in our models lie above the exclusion limits.

The $\tilde{\rho}$ which couple to SM fermions via (3.4) appear as resonances in dijet and/or dilepton channels. However, the $\tilde{\rho}$ decay to $\tilde{\pi} \tilde{\pi}$ (3.5) completely dominates over the decays to SM fermion pairs (3.7). The branching fraction to SM fermions is largest for the $\tilde{\rho}$ which mixes with a gluon, which therefore can decay to quarks. Even in that case, however, the Tevatron dijet resonance searches [30] cannot exclude such a $\tilde{\rho}$, as shown in [4]. As for dileptons, the fact that it has to go through a $\tilde{\rho}$ that mixes with a $Z$ or $\gamma$ reduces the cross section, and together with the small branching fraction, this is easily consistent with Tevatron resonance searches [23, 31, 34].

\subsection{Summary}

Before we go on to illustrate some of the generic phenomenological features of Vectorlike Confinement, we want to summarize the types of processes generated by the effective Lagrangian in (3.1). At the LHC, hyper- $\rho$ 's can be resonantly produced from a $q-\bar{q}$ initial state and decay to a pair of hyperpions, which is illustrated in figure 2. The $\tilde{\rho}$ that can be resonantly produced can be thought of as massive copies of the SM gauge bosons (photons, $W$ 's, $Z$ 's or gluons). Note that the hyperpions that are charged under the SM will also be pair produced through Drell-Yan processes, in fact this is in general the dominant production mechanism. Once produced, the short-lived hyperpions will decay promptly to a pair of SM gauge bosons while the long-lived hyperpions will generically pass through the detector without decaying, in which case they will appear as CHAMP's and Rhadrons, or may be invisible in some cases. It is however possible for the long-lived pions to decay 

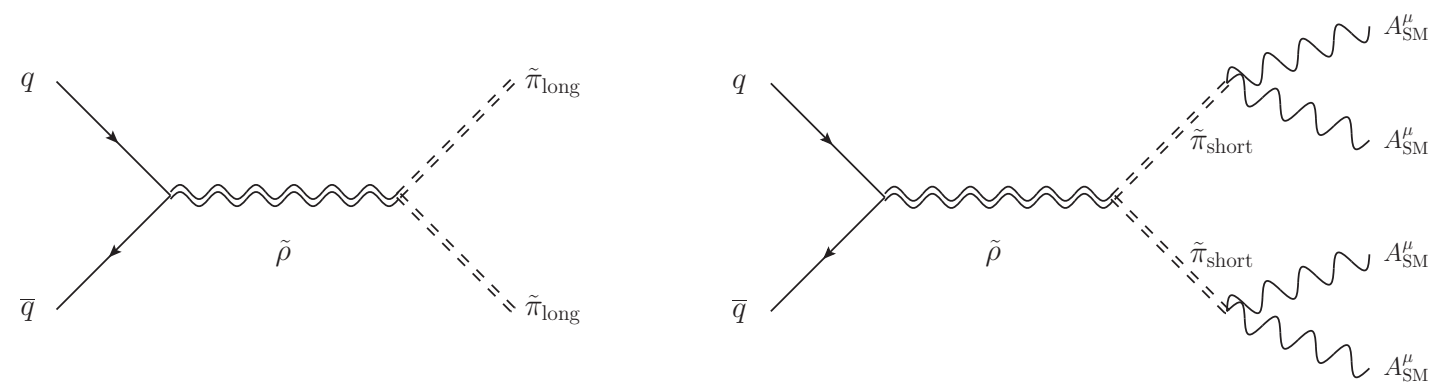

Figure 2: The resonant production of hyper- $\rho$ 's and their subsequent decays to a pair of hyperpions. If the hyperpions are of the short-lived type they will decay promptly to a pair of SM gauge bosons whereas if they are of the long-lived type they will register as long-lived massive charged/colored particles.

to SM fermion pairs within the detector if the terms in (3.16) leading to their decay incorporate a nontrivial flavor structure.

\section{Phenomenological Highlights}

In this section, we would like to go through various phenomenological features that generically arise in Vectorlike Confinement. In particular, the existence of short-lived and long-lived pseudoGoldstones leads to multiple gauge bosons and collider stable charged particles in the final state, respectively. Depending on whether the long-lived particles carry SU(3) color charge or not, they are classified as R-hadrons or CHAMPs, respectively. They are always produced in pairs, and when they are created by the decay of a $\tilde{\rho}$, it becomes possible to reconstruct the primary resonance, which is a novel signature.

It is possible for the long-lived $\tilde{\pi}$ to still decay within collider timescales if the nonrenormalizable interactions that allow them to decay have a relatively low scale, which can lead to narrow resonances of various SM fermion pairs such as dileptons, diquarks or leptoquarks. (Recall that this is only possible with an extra flavor structure in the coefficients appearing in (3.16).) Finally, if one (or more) of the SM singlet $\tilde{\pi}$ is extremely long-lived or exactly stable, it is a (possibly decaying) dark matter candidate.

For each signature type we will choose a simple model of Vectorlike Confinement in which they occur, but more intricate setups are certainly possible. For each model we discuss, we will also briefly comment on how hyperbaryons can decay. Hyperbaryon phenomenology depends strongly on the number of hypercolors and we will not attempt to cover all possibilities in complete generality. Instead, for each model we discuss, we will explicitly write down an operator for a specific choice of the hypercolor group that violates hyperbaryon number so its inclusion in the Lagrangian can lead to hyperbaryon decay. 


\subsection{Multiple Gauge-boson Production}

Except for the case of all $\Psi$ being SM singlets, Vectorlike Confinement phenomenology always includes one (or more) $\tilde{\rho}$ that mixes with a SM gauge boson as well as a $\tilde{\pi}$ in the same SM representation. Such a $\tilde{\pi}$ decays promptly to a pair of SM gauge bosons via (3.1d). Therefore, for any SM charge assignments of the $\Psi$, when such a $\tilde{\rho}$ is resonantly produced from a $q-\bar{q}$ initial state via (3.4), one of its decay modes is always to a pair of $\tilde{\pi}$ which subsequently decay to four SM gauge bosons. This final state is robust in all models of Vectorlike Confinement.

Such a signature with four gluons was studied in [4 for the 3-flavor, 1-species model with $\Psi \sim(\mathbf{3}, \mathbf{3})$ under $\mathrm{SU}(3)_{\mathrm{HC}} \otimes \mathrm{SU}(3)_{\mathrm{C}}$. In this case there is no coupling to leptons since the hypersector couple to color only, and the $\tilde{\rho}$, which mixes with a gluon, can be as light as $350 \mathrm{GeV}$, and has been shown to be discoverable in existing Tevatron data.

Naively, one might think that an analogous model for electroweak interaction, i.e. the 2-flavor 1-species model with $\Psi \sim(\mathbf{N}, 2)$ under $\mathrm{SU}(N)_{\mathrm{HC}} \otimes \mathrm{SU}(2)_{\mathrm{W}}$, should give signatures with four electroweak gauge bosons. However, this is incorrect for a subtle reason specific to this model, which we discuss in Appendix A. The signature of multiple electroweak gauge boson production is retained if one adds one more hyperflavor to the model, which is the case we will discuss next. Since this addition also causes the existence of a di-CHAMP resonance, we will investigate both types of signatures in the next subsection Sec. 4.2.

\subsection{A Model of multi EW-boson Production and Di-CHAMP Resonances}

In order to have a long-lived $\tilde{\pi}$ in the spectrum, the hyperflavors need to arrange themselves into at least two species. For this purpose we will choose to work with the following model with 3 hyperflavors which arrange themselves into 2 -species, a weak doublet and a singlet with hypercharge:

\begin{tabular}{c|c||c|c|c} 
& $\mathrm{SU}(N)_{\mathrm{HC}}$ & $\mathrm{SU}(3)_{\mathrm{C}}$ & $\mathrm{SU}(2)_{\mathrm{W}}$ & $\mathrm{U}(1)_{\mathrm{Y}}$ \\
\hline$\Psi_{\mathbf{2}}$ & $\square$ & $\mathbf{1}$ & $\square$ & $-1 / 2$ \\
$\Psi_{\mathbf{1}}$ & $\square$ & $\mathbf{1}$ & $\mathbf{1}$ & 1
\end{tabular}

Note that $\Psi_{1,2}$ are Dirac fermions.

\subsubsection{Spectrum}

Let us begin by listing the states in the spectrum. In order to display the quantum numbers of each state we will use the notation $\left(r_{\mathrm{SU}(3)_{\mathrm{C}}}, r_{\mathrm{SU}(2)_{\mathrm{W}}}\right)_{\mathrm{U}(1)_{\mathrm{Y}}}$. The $\tilde{\rho}$ which mix with SM gauge bosons are

$$
\begin{aligned}
\left(W^{\prime 0}, W^{\prime \pm}\right) & \sim(\mathbf{1}, \mathbf{3})_{0} \\
B^{\prime} & \sim(\mathbf{1}, \mathbf{1})_{0}
\end{aligned}
$$

while the rest of $\tilde{\rho}$ transform as

$$
\begin{aligned}
\left(\tilde{\rho}^{++}, \tilde{\rho}^{+}\right) & \sim(\mathbf{1}, \mathbf{2})_{3 / 2} \\
\tilde{\rho}_{0}^{\prime \prime} & \sim(\mathbf{1}, \mathbf{1})_{0}
\end{aligned}
$$




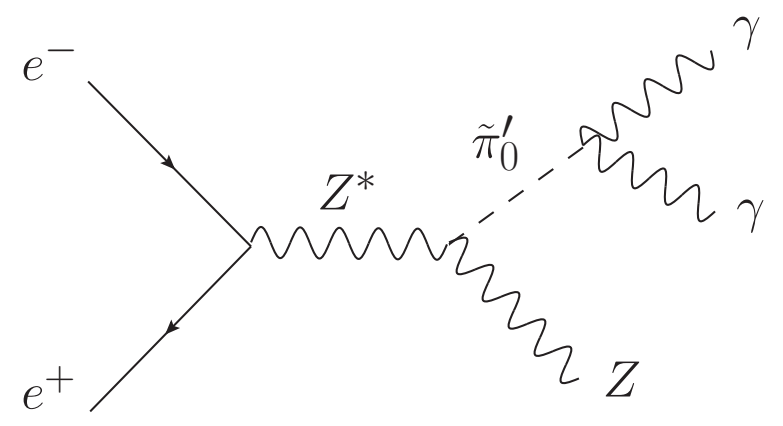

Figure 3: Emission of an $\tilde{\pi}^{0}$ in a Drell-Yan process and subsequent decay to a pair of photons is found to be consistent with low mass Higgs boson searches at LEP.

where the $\tilde{\rho}_{0}^{\prime \prime}$ corresponds to the hyperbaryon $\mathrm{U}(1)_{\mathrm{HB}}$ current. The $\tilde{\pi}$ 's fill in the following multiplets:

$$
\begin{aligned}
\left(\tilde{\pi}^{0}, \tilde{\pi}^{ \pm}\right) & \sim(\mathbf{1}, \mathbf{3})_{0}, \\
\left(\widetilde{K}^{++}, \widetilde{K}^{+}\right) & \sim(\mathbf{1}, \mathbf{2})_{3 / 2}, \\
\tilde{\pi}_{0}^{\prime} & \sim(\mathbf{1}, \mathbf{1})_{0} .
\end{aligned}
$$

The $\tilde{\pi}$ masses from SM gauge loops (3.9) before EWSB are

$$
\begin{aligned}
m_{\tilde{\pi}^{0}, \tilde{\pi}^{ \pm}} & \simeq 0.13 \mathrm{~cm} m_{\rho}, \\
m_{\widetilde{K}^{++}, \widetilde{K}^{+}} & \simeq 0.11 c m_{\rho},
\end{aligned}
$$

where the coefficient $c$ was defined in equation (3.9) and has the value 1.1 for $N=3=F$ as we know from QCD. The SM-singlet $\tilde{\pi}_{0}^{\prime}$ does not get a mass from SM gauge loops, so $m_{\tilde{\pi}_{0}^{\prime}}$ arises entirely from hyperquark masses (3.10). After EWSB, the $\tilde{\pi}^{ \pm}-\tilde{\pi}^{0}$ and $\widetilde{K}^{++}-\widetilde{K}^{+}$mass splittings are given by (3.11) as

$$
\begin{aligned}
m_{\tilde{\pi}^{ \pm}}-m_{\tilde{\pi}^{0}} & \approx 170 \mathrm{MeV} \\
m_{\widetilde{K}^{++}}-m_{\widetilde{K}^{+}} & \approx 1.1 \mathrm{GeV} .
\end{aligned}
$$

\subsubsection{Model specific constraints}

The SM-singlet $\tilde{\pi}_{0}^{\prime}$ shares many properties with axions, such as the coupling to two photons via $\tilde{\pi}_{0}^{\prime} F \tilde{F}$ from (3.1d). Unlike the ordinary Peccei-Quinn axion, however, its couplings to SM fermions are induced only at two-loop level or via nonrenormalizable operators that couple hyperquarks to SM fermions. (It still couples to nucleons with a similar strength as the ordinary axions because it mixes with a $\pi^{0}$ ). The two-loop suppression, however, is not enough to evade astrophysical bounds such as supernova cooling if $m_{\tilde{\pi}_{0}^{\prime}} \lesssim 100 \mathrm{MeV}$. On the other hand, it is enough to account for the absence $\left(\lesssim 10^{-10}[21]\right)$ of the decays $K^{+} \rightarrow \pi^{+} A^{0} \rightarrow \pi^{+} e^{+} e^{-}$or $\rightarrow \pi^{+} \mu^{+} \mu^{-}$, where $A^{0}$ is an axion, because the two-loop suppression factor enters twice in the amplitudes of these processes. Therefore, we take $m_{\tilde{\pi}_{0}^{\prime}}$ to be at least $\sim \mathcal{O}(100) \mathrm{MeV}$, corresponding to hyperquark masses of $\gtrsim \mathcal{O}(10) \mathrm{keV}$ for $m_{\tilde{\rho}} \sim \mathrm{TeV}$. Then the constraints from beam-dump experiments which looked for 
axion-like particles are also evaded $[35] 11$.

If $m_{\tilde{\pi}_{0}^{\prime}}<10 \mathrm{GeV}$, a $\Upsilon$ can decay into a $\gamma$ and a $\tilde{\pi}_{0}^{\prime}$ which then decays promptly to two $\gamma$ 's. This could in principle be observable at BaBar [37], but since the $\tilde{\pi}_{0}^{\prime}-\gamma-\gamma$ vertex (3.1d) is one-loop suppressed, it only amounts to less than one event. Similarly, since the $\tilde{\pi}_{0}^{\prime}-Z-Z$ coupling is also one-loop suppressed, the production of a $\tilde{\pi}_{0}^{\prime}$ in association with a $Z$ at lepton colliders (see Fig. 3) is highly suppressed, and therefore falls way below the bounds from the LEP Higgs searches in the similar channel [38].

\subsubsection{Decays and collider phenomenology}

Let us now discuss the phenomenology of the $\tilde{\rho}$ and non-SM-singlet $\tilde{\pi}$. In colliders, a $W^{\prime}$ can be resonantly produced from a $q-\bar{q}$ initial state, and will dominantly decay via (3.1c) to

$$
\begin{array}{rll}
W^{\prime 0} & \stackrel{2 / 3}{\longrightarrow} & \tilde{\pi}^{+}+\tilde{\pi}^{-} \\
& \stackrel{1 / 6}{\longrightarrow} & \widetilde{K}^{++}+\widetilde{K}^{--} \\
& \stackrel{1 / 6}{\longrightarrow} & \widetilde{K}^{+}+\widetilde{K}^{-}, \\
W^{\prime+} & \stackrel{2 / 3}{\longrightarrow} & \tilde{\pi}^{0}+\tilde{\pi}^{+} \\
& \stackrel{1 / 3}{\longrightarrow} & \widetilde{K}^{++}+\widetilde{K}^{-} .
\end{array}
$$

Similarly, a $B^{\prime}$ can also be resonantly produced and dominantly decays via (3.1c) as

$$
\begin{aligned}
& B^{\prime} \stackrel{1 / 2}{\longrightarrow} \widetilde{K}^{++}+\widetilde{K}^{--} \\
& \stackrel{1 / 2}{\longrightarrow} \widetilde{K}^{+}+\widetilde{K}^{-} .
\end{aligned}
$$

(The branching fractions above are approximate as the differences of the hyperpion masses are ignored.)

The hyperpions can be produced from Drell-Yan processes or through the decays above. The $\tilde{\pi}^{ \pm}$and $\tilde{\pi}^{0}$ decay promptly to electroweak gauge bosons via the vertex (3.1d):

$$
\begin{aligned}
& \tilde{\pi}^{ \pm} \longrightarrow W^{ \pm} Z, W^{ \pm} \gamma, \\
& \tilde{\pi}^{0} \longrightarrow 2 Z, Z \gamma, 2 \gamma .
\end{aligned}
$$

(These are actually $W^{ \pm} B$ and $W^{3} B$, respectively.) Since the branching fractions of these decays have only a mild dependence on phase space, we take a representative value 12 of $m_{\tilde{\pi}}=355 \mathrm{GeV}$ and find that the $\tilde{\pi}^{ \pm}$decays to $W \gamma 81 \%$ of the time and to $W Z 19 \%$ of the time, while the branching fractions of the $\tilde{\pi}^{0}$ to $\gamma \gamma, \gamma Z$ and $Z Z$ are given by $43 \%, 29 \%$ and $28 \%$ respectively. We therefore have a very interesting collider phenomenology with little background. Since these $\tilde{\pi}$ are pair-produced where each in turn decays to a pair of SM gauge bosons, there will be a significant fraction of events with multiple leptons as well as photons in the final state. In particular, the $\tilde{\pi}^{0}$ pair-production will give rise to $4 \gamma$ events which can be paired up to reveal the $\tilde{\pi}^{0}$ resonances. Similarly, the $\tilde{\pi}^{ \pm}$pair production can lead to $\ell^{+} \ell^{-} \gamma \gamma+$ missing $E_{\mathrm{T}}$.

\footnotetext{
${ }^{11}$ A recent interest in studying "dark" U(1) gauge bosons has also led to a reanalysis of these beam-dump experiments [36] in terms of parameters that are better suited for a light vector particle rather than an axion. Future experiments proposed in [36] will almost certainly improve the bounds on $\tilde{\pi}_{0}^{\prime}$ as well.

${ }^{12}$ This corresponds to $m_{\tilde{\rho}}=2.5 \mathrm{TeV}$ which we will use in Fig. 4]
} 
In contrast, the $\widetilde{K}$ carry nonzero species numbers and thus do not have the couplings (3.1d) to gauge bosons. Instead, because of the mass splitting (4.7), a $\widetilde{K}^{++}$can decay to a $\widetilde{K}^{+}$, which is lighter and carries the same species numbers:

$$
\begin{aligned}
\widetilde{K}^{++} \longrightarrow & \widetilde{K}^{+}+\pi^{+}, \quad \widetilde{K}^{+}+K^{+}, \\
& \widetilde{K}^{+}+e^{+}+\nu_{e}, \quad \widetilde{K}^{+}+\mu^{+}+\nu_{\mu} .
\end{aligned}
$$

These decays are mediated by an off-shell $W$ boson, and the rates are given by

$$
\begin{aligned}
\Gamma_{\widetilde{K}^{++} \rightarrow \widetilde{K}^{+}+\pi^{+}}^{-1} & =220 \mu \mathrm{m}\left(\frac{1.1 \mathrm{GeV}}{\Delta m_{\widetilde{K}}}\right)^{3} \sqrt{\frac{1-m_{\pi^{+}}^{2} /(1.1 \mathrm{GeV})^{2}}{1-m_{\pi^{+}}^{2} / \Delta m_{\widetilde{K}}^{2}}} \\
\Gamma_{\widetilde{K}^{++} \rightarrow \widetilde{K}^{+}+K^{+}}^{-1} & =4.1 \mathrm{~mm}\left(\frac{1.1 \mathrm{GeV}}{\Delta m_{\widetilde{K}}}\right)^{3} \sqrt{\frac{1-m_{K^{+}}^{2} /(1.1 \mathrm{GeV})^{2}}{1-m_{K^{+}}^{2} / \Delta m_{\widetilde{K}}^{2}}}
\end{aligned}
$$

and

$$
\begin{aligned}
\Gamma_{\widetilde{K}^{++} \rightarrow \widetilde{K}^{+}+e^{+}+\nu_{e}}^{-1} & =480 \mu \mathrm{m}\left(\frac{1.1 \mathrm{GeV}}{\Delta m_{\widetilde{K}}}\right)^{5} \\
& \simeq \Gamma_{\widetilde{K}^{++} \rightarrow \widetilde{K}^{+}+\mu^{+}+\nu_{\mu}}^{-1} .
\end{aligned}
$$

Unfortunately, even though these rates are of the right size for displaced vertices, the outgoing $\pi^{ \pm}$, $e^{ \pm}, \mu^{ \pm}$are all too soft to be seen in detectors. The bright side of this fact is that since the energy of the parent $\widetilde{K}^{ \pm \pm}$is almost entirely carried away by the daughter $\widetilde{K}^{ \pm}$, a $\widetilde{K}^{ \pm \pm}$can practically be identified as a $\widetilde{K}^{ \pm}$in collider experiments, effectively increasing the $\widetilde{K}^{ \pm}$production rate.

On the other hand, the $\widetilde{K}^{ \pm}$is the lightest particle with nonzero species numbers, and therefore can only decay via a non-renormalizable operator that violate species numbers, as discussed in Sec. 2.2.2. The leading such operator is given by the current-current operator (2.10) with $\left(\Psi_{J}, \Psi_{I}, f_{i}, f_{j}\right)=\left(\Psi_{1}, \Psi_{2}, e_{i}^{c}, \ell_{j}\right)$. This operator gives rise to

$$
\begin{gathered}
\widetilde{K}^{+} \longrightarrow \bar{\ell}_{i}+\nu_{j} \\
\widetilde{K}^{++} \longrightarrow \bar{\ell}_{i}+\bar{\ell}_{j},
\end{gathered}
$$

with the rates given by (3.17). Therefore, it is quite likely that $\widetilde{K}^{ \pm}$is collider stable (unless a nontrivial flavor structure is present in (3.17) to allow for significantly lower $M$ ), while the $\widetilde{K}^{ \pm \pm}$ width is dominated by the weak decays (4.11).

Thus, we expect a novel collider signature, a "di-CHAMP resonance," where it is possible to reconstruct the parent $W^{\prime 0}$ or $B^{\prime}$ from a $\widetilde{K}^{+}-\widetilde{K}^{-}$pair. Here recall that a $\widetilde{K}^{ \pm \pm}$cannot be distinguished from a $\widetilde{K}^{ \pm}$, so $W^{\prime 0}$ and $B^{\prime}$ decays to $\widetilde{K}^{ \pm \pm}$as well as $W^{\prime \pm}$ production also contribute to the signal. Since CHAMPs have no irreducible backgrounds, we do not need a large luminosity for CHAMP analysis. Note, however, that the CHAMPs can also be produced through Drell-Yan processes, which tends to be larger than the production through a $\tilde{\rho}$ resonance, due to the large mass of the $\tilde{\rho}$. In Fig. 4, we plot the CHAMP production cross section as a function of the pair invariant mass at the $\operatorname{LHC}(\sqrt{s}=14 \mathrm{TeV})$, with the number of hypercolors $N=3$ (thus taking $c=1.1$ in (4.5) $)$ for $m_{\tilde{\rho}}=2.5 \mathrm{TeV}\left(m_{\widetilde{K}}=3.0 \times 10^{2} \mathrm{GeV}\right)$ and $m_{\tilde{\rho}}=4.0 \mathrm{TeV}\left(m_{\widetilde{K}}=4.8 \times 10^{2}\right.$ $\mathrm{GeV}$ ). For this plot, the parameter values $g_{\tilde{\rho} \tilde{\pi} \tilde{\pi}}=6, \varepsilon_{W^{\prime}}=0.1$ and $\varepsilon_{B^{\prime}}=0.1$ were used. (Note 


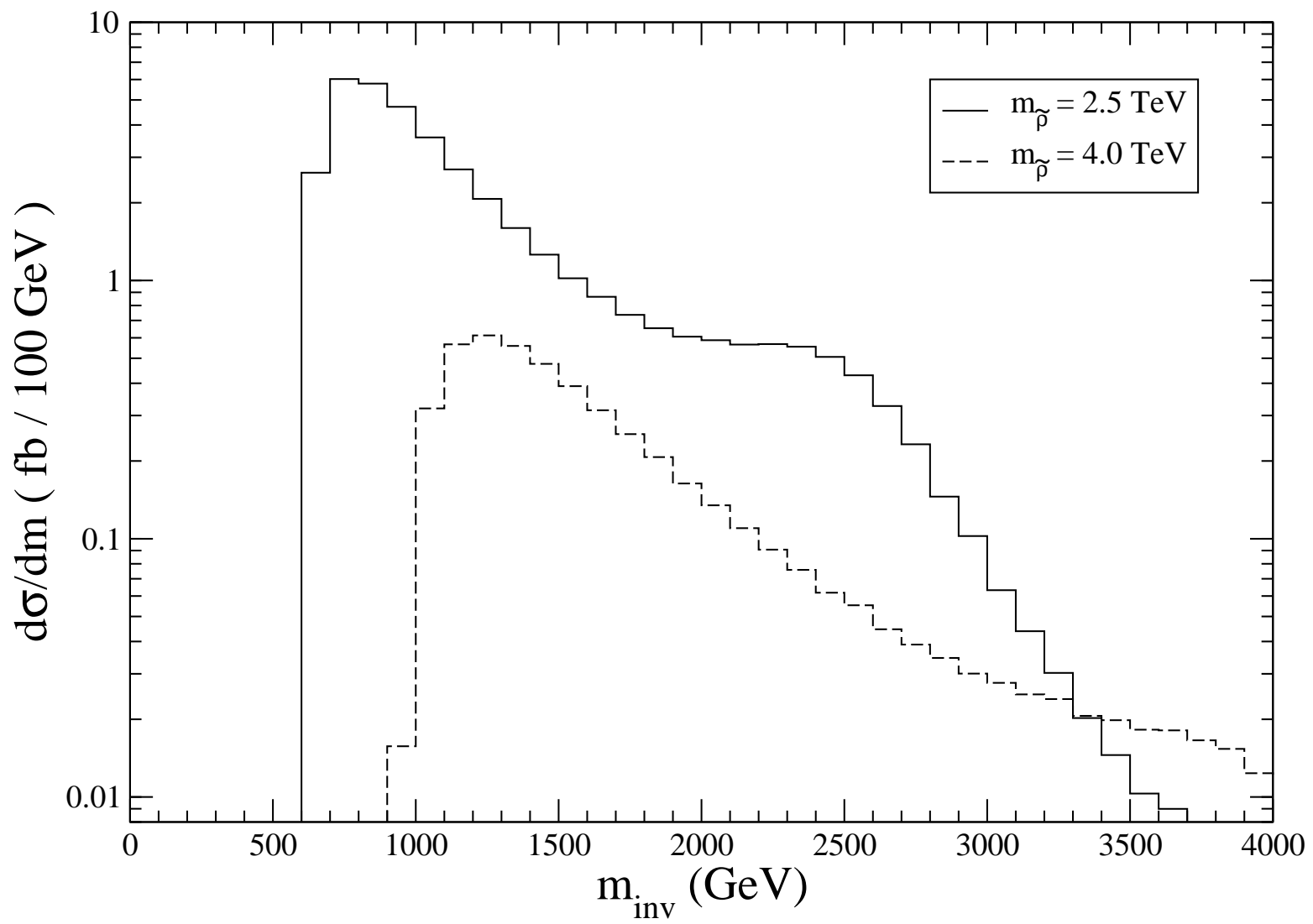

Figure 4: The differential cross section for the pair production of CHAMPs for $\sqrt{s}=14 \mathrm{TeV}$, $m_{\tilde{\rho}}=2.5 \mathrm{TeV}$ and $m_{\tilde{\rho}}=4.0 \mathrm{TeV}$ at the LHC. While most of the CHAMPs come from Drell-Yan processes, at high enough energies resonant $\tilde{\rho}$ production becomes the dominant source.

that the latter includes a factor of $\sqrt{3}$ due to the normalization of SM hypercharge relative to the corresponding hyperflavor generator $T^{8}$.) One sees that even though most events are induced by Drell-Yan processes, there is enough statistics in the lighter case for the parent $\tilde{\rho}$ resonance to be clearly noticeable, given that there is no irreducible background. Furthermore, it is possible to analyze the angular distribution of the CHAMP pairs and see that the primary resonance is indeed a spin-1 particle decaying to a pair of scalars. In particular, one can do this by keeping only the high invariant mass events, subtracting the Drell-Yan "background", which has the same angular distribution.

In principle, there is also a possibility of pair-producing $\tilde{\rho}$ 's. Note, however, that the large mass gap (4.5) between the $\tilde{\pi}$ and $\tilde{\rho}$ implies $m_{\tilde{\rho}} \sim$ a few TeV. Having only electroweak gauge interactions, a pair of $\tilde{\rho}$ 's in this mass range is challenging at the LHC.

Finally, all CHAMP and R-hadron searches at the Tevatron [39,40] limit the production crosssection of the $\tilde{\pi}$ to be less than about $0.1 \mathrm{pb}$. As in the LHC case above, they are dominantly produced by SM gauge interactions, and the $0.1 \mathrm{pb}$ bound on the cross section translates to a lower bound on the CHAMP mass of about $250-300 \mathrm{GeV}$. 


\subsubsection{Hyperbaryons}

Before moving on to the next model, we would like to comment briefly on hyperbaryon decays in this model. In the specific case where $N_{\mathrm{HC}}=4$, one can include in the Lagrangian the following operator (among others) that violates hyperbaryon number:

$$
\mathcal{O}_{\mathrm{HB}}=\epsilon^{A B C D}\left(\Psi_{\mathbf{2} A}^{\mathrm{T}} \mathcal{C} \Psi_{\mathbf{2} B}\right)\left(h \Psi_{\mathbf{2} C}^{\mathrm{T}} \mathcal{C} \Psi_{\mathbf{1} D}\right)+\text { h.c. }
$$

where the uppercase letters denote hypercolor indices and $\mathcal{C}$ is the charge conjugation matrix. The $\mathrm{SU}(2)_{\mathrm{W}}$ indices are implicitly contracted within each bracket, between a pair of $\Psi_{2}$ 's or a $\Psi_{2}$ and a SM Higgs. One can check that the hypercharges add to zero. $\mathcal{O}_{\mathrm{HB}}$ can lead to the decay of a hyperbaryon to a number of hyperpions and a component of the Higgs doublet.

For different choices of $N_{\mathrm{HC}}$ one can write down similar operators by replacing some of the $\Psi$ 's by a judicious choice of SM fermions and adding Higgs insertions such that all SM gauge indices can be contracted. Note that the mass dimension of the operator will in general depend on $N_{\mathrm{HC}}$.

\subsection{A Model of Di-R-hadron Resonances}

The appearance of R-hadrons in Vectorlike Confinement theories is quite generic, a necessary and sufficient condition being just the existence of two (or more) species, at least one of which carries QCD color. In order to illustrate the relevant phenomenology we choose the following minimal model with four flavors that arrange themselves into two species, one color triplet and a singlet with hypercharge:

\begin{tabular}{c|c||c|c|c} 
& $\mathrm{SU}(N)_{\mathrm{HC}}$ & $\mathrm{SU}(3)_{\mathrm{C}}$ & $\mathrm{SU}(2)_{\mathrm{W}}$ & $\mathrm{U}(1)_{\mathrm{Y}}$ \\
\hline$\Psi_{\mathbf{3}}$ & $\square$ & $\square$ & $\mathbf{1}$ & $-1 / 3$ \\
$\Psi_{\mathbf{1}}$ & $\square$ & $\mathbf{1}$ & $\mathbf{1}$ & 1
\end{tabular}

Note that $\Psi_{1,3}$ are Dirac fermions.

\subsubsection{Spectrum}

The $\tilde{\rho}$ and $\tilde{\pi}$ spectra are as follows. The $\tilde{\rho}$ which mix with SM gauge bosons are

$$
\begin{aligned}
g^{\prime} & \sim(\mathbf{8}, \mathbf{1})_{0}, \\
B^{\prime} & \sim(\mathbf{1}, \mathbf{1})_{0},
\end{aligned}
$$

while the rest of $\tilde{\rho}$ 's transform as

$$
\begin{aligned}
& \tilde{\rho}_{\mathbf{3}} \sim(\mathbf{3}, \mathbf{1})_{-4 / 3}, \\
& \tilde{\rho}_{0}^{\prime \prime} \sim(\mathbf{1}, \mathbf{1})_{0},
\end{aligned}
$$

where the $\tilde{\rho}_{0}^{\prime \prime}$ corresponds to the hyperbaryon $\mathrm{U}(1)_{\mathrm{HB}}$ current. The $\tilde{\pi}$ fill in the following multiplets.

$$
\begin{aligned}
& \tilde{\pi}_{\mathbf{8}} \sim(\mathbf{8}, \mathbf{1})_{0}, \\
& \tilde{\pi}_{\mathbf{3}} \sim(\mathbf{3}, \mathbf{1})_{-4 / 3}, \\
& \tilde{\pi}_{0}^{\prime} \sim(\mathbf{1}, \mathbf{1})_{0} .
\end{aligned}
$$


The $\tilde{\pi}$ masses from SM gauge loops (3.9) are

$$
\begin{aligned}
& m_{\tilde{\pi}_{8}} \simeq 0.29 c m_{\tilde{\rho}}, \\
& m_{\tilde{\pi}_{3}} \simeq 0.20 c m_{\tilde{\rho}},
\end{aligned}
$$

while the singlet $\tilde{\pi}_{0}^{\prime}$ does not get a mass from SM gauge loops, so $m_{\tilde{\pi}_{0}^{\prime}}$ arises entirely from hyperquark masses (3.10).

\subsubsection{Model specific constraints}

As we discussed in section 4.2.2, we take $m_{\tilde{\pi}_{0}^{\prime}} \gtrsim \mathcal{O}(100) \mathrm{MeV}$. But unlike the $\tilde{\pi}_{0}^{\prime}$ there, the $\tilde{\pi}_{0}^{\prime}$ here not only couples to two photons but also to two gluons via (3.1d). Thus, a $\tilde{\pi}_{0}^{\prime}$ can be resonantly produced from $g g$ at hadron colliders, which subsequently decays to $g g(\approx 99 \%)$ or $\gamma-\gamma(\approx 1 \%)$. Just like the $g g \rightarrow \tilde{\pi}_{\mathbf{8}} \rightarrow g g$ case discussed in [4], the $g g \rightarrow \tilde{\pi}_{0}^{\prime} \rightarrow g g$ dijet process here is completely overwhelmed by QCD dijet processes, due to the one-loop suppression in the vertex (3.1d). On the other hand, the $g g \rightarrow \tilde{\pi}_{0}^{\prime} \rightarrow \gamma \gamma$ could be clean enough, despite its low branching fraction. At the Tevatron, inclusive di-photon searches have been performed [41, but it turns out that the $\tilde{\pi}_{0}^{\prime}$ is not ruled out for $m_{\tilde{\pi}_{0}^{\prime}}<100 \mathrm{GeV}$. (We will not consider $m_{\tilde{\pi}_{0}^{\prime}} \gtrsim 100 \mathrm{GeV}$, as this cannot be obtained from hyperquark masses $\ll m_{\tilde{\rho}}$.) See Appendix $\mathrm{B}$ for the detail of the analysis. Finally, at Babar, a $\Upsilon$ can decay into a photon and a $\tilde{\pi}_{0}^{\prime}$ which subsequently decays promptly to two gluons. While this dominates over the two-photon mode discussed in Sec. 4.2, it would be completely buried under SM backgrounds.

\subsubsection{Decays and collider phenomenology}

Let us move on to the phenomenology of the $\tilde{\rho}$ and the non-singlet $\tilde{\pi}$. The $g^{\prime}$ and $B^{\prime}$ can be produced resonantly at colliders and decay via (3.1c):

$$
\begin{aligned}
& g^{\prime} \stackrel{3 / 4}{\longrightarrow} \quad \tilde{\pi}_{\mathbf{8}}+\tilde{\pi}_{\mathbf{8}} \\
& \stackrel{1 / 4}{\longrightarrow} \tilde{\pi}_{3}+\overline{\tilde{\pi}_{3}}, \\
& B^{\prime} \longrightarrow \tilde{\pi}_{3}+\overline{\tilde{\pi}_{3}} \text {, }
\end{aligned}
$$

(These branching fractions are approximate as the $\tilde{\pi}$ mass differences are ignored.)

The $\tilde{\pi}_{\mathbf{8}}$ decays promptly via (3.1d) as

$$
\tilde{\pi}_{\mathbf{8}} \longrightarrow 2 g, g Z, g \gamma \text {. }
$$

The $\tilde{\pi}_{3}$ requires a nonrenormalizable operator to decay, as it carries nonzero species number. The leading operator for $\tilde{\pi}_{3}$ decay is given by the "scalar-scalar" operator (2.11) with $\left(\Psi_{J}, \Psi_{I}, f_{i}, f_{j}\right)=$ $\left(\Psi_{1}, \Psi_{3}, e_{i}^{c}, d_{j}^{c}\right)$. This induces the decay

$$
\tilde{\pi}_{3} \longrightarrow \ell_{i}+d_{j}
$$

with the rate given by (3.18). Therefore, we expect that the $\tilde{\pi}_{3}$ is collider stable (unless an extra flavor symmetry is introduced to significantly lower $M$ ). Once produced, it will hadronize rapidly into an R-hadron, sometimes neutral and sometimes charged, in roughly equal numbers. If both 


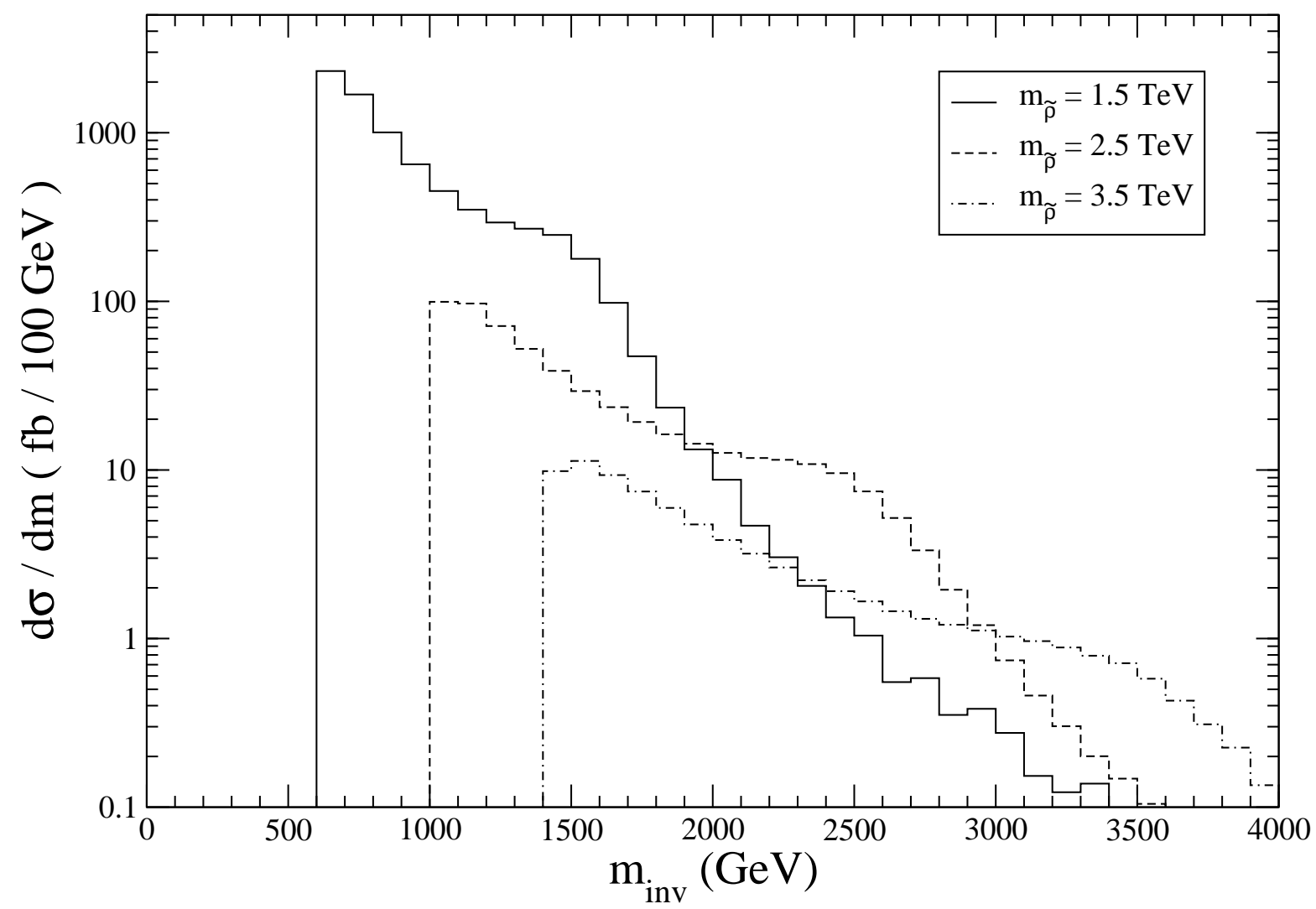

Figure 5: The differential cross section for the pair production of R-hadrons at the LHC $(\sqrt{s}=14)$ $\mathrm{TeV}$ for three choices of $m_{\tilde{\rho}}$ corresponding to an $m_{\tilde{\pi}_{3}}$ of 300,500 and $700 \mathrm{GeV}$. At high energies, decays of resonantly produced $\tilde{\rho}$ dominate the production.

R-hadrons from a $g^{\prime}$ or $B^{\prime}$ decay end up charged, then we again have a resonance in a pair of long lived charged particles. Although the $\tilde{\rho}$ width in this case is slightly larger than the case of Sec. 4.2 due to larger $F$ in (3.5) ), the resonant $\tilde{\rho}$ can now be lighter, since $m_{\tilde{\pi}} / m_{\tilde{\rho}}$ from (3.9) is larger. Therefore, we expect a larger cross section for the resonant production of $\tilde{\pi}_{3}$ 's than that of $\widetilde{K}^{ \pm}$'s in Sec. 4.2, which is illustrated in figure 5. In this plot the parameter values $g_{\tilde{\rho} \tilde{\pi} \tilde{\pi}}=6, c=1$ and $\varepsilon_{g}=0.2$ were used. Again, it should be interesting to analyze the angular distribution of the $\mathrm{R}$-hadron pairs and observe that the primary resonance is indeed spin-1.

Note that for a given $\mathrm{m}_{\tilde{\pi}}, m_{\tilde{\rho}}$ can be lighter compared to the previous model, so that pairproducing $g^{\prime}$ 's and $\tilde{\rho}_{3}$ 's might be interesting as well. Such pair-production of $g^{\prime}$ was shown to be discoverable in the multijet channel [5]. Including the R-hadrons in the decay products can make the signal quite spectacular, with the production of 4 R-hadrons with an $\mathcal{O}(\mathrm{fb})$ cross-section.

\subsubsection{Hyperbaryons}

Similar to the model presented previously, we can write down a Lorentz invariant, gauge singlet operator that allows the hyperbaryons to decay in this model. Once again, we content ourselves with a specific example for the choice of the hypercolor group, in particular for $N=4$ one can 
write down

$$
\mathcal{O}_{\mathrm{HB}}=\epsilon^{A B C D}\left(\Psi_{\mathbf{3} A}^{\mathrm{T}} \mathcal{C} \Psi_{\mathbf{3} B}\right)\left(\Psi_{\mathbf{3} C}^{\mathrm{T}} \mathcal{C} \Psi_{\mathbf{1} D}\right)+\text { h.c. }
$$

where the suppressed $\mathrm{SU}(3)_{\mathrm{C}}$ indices of the $\Psi_{\mathbf{3}}$ are contracted with an $\epsilon^{i j k}$. This operator breaks hyperbaryon number and including it in the Lagrangian makes it possible for hyperbaryons to decay into a number of $\tilde{\pi}$. For different choices of $N_{\mathrm{HC}}$, one can trade $\Psi$ 's for SM fermions (or add Higgs insertions) in order to make a gauge singlet.

\subsection{Leptoquarks, Dileptons, and Diquarks (with Displaced Vertices)}

Another collider signature that can arise in a variety of Vectorlike Confinement models is that of narrow resonances in pairs of SM fermions. As we discussed in Sec. 2.3.1, if extra flavor structures are imposed on the mixed SM-hyper 4-fermion operators (2.10) and (2.11), it is possible to lower the scale $M$ so that long-lived $\tilde{\pi}$ decay within the detector, possibly with displaced vertices.

For example, the $\widetilde{K}^{ \pm}$CHAMP of Sec. 4.2 can decay inside the detector to $\tau^{+} \nu_{\tau}$. (Note the strong preference of the rate (3.17) for heavier flavors.) Similarly, $\widetilde{K}^{++}$will promptly decay to $\tau^{+} \tau^{+}$, beating the rate (4.11). Therefore, the $\widetilde{K}^{++}-\widetilde{K}^{--}$pair production, either from Drell-Yan or from the $W^{\prime 0}$ decay, will dominantly end up in $4 \tau$ 's. These $\tau$ 's are highly boosted, hence difficult to identify, but it may help to utilize the leptonic $\tau$ decays.

Similarly, if $M$ is sufficiently lowered, the $\tilde{\pi}_{\mathbf{3}}$ R-hadron of Sec. 4.3 will decay inside the detector to a charged lepton and a $d$-type quark, hence behaving as a leptoquark, offering an exciting collider signature. Although the 4 -fermion operator responsible for the $\tilde{\pi}_{\mathbf{3}}$ decay is of the "scalarscalar" form (2.11), we again expect that the rate (3.18) would prefer decays to the 3rd generation, because flavor violation constraints requires that this operator should be small for the 1st and 2nd generations. The Tevatron has set the lower limits on the mass of such "3rd generation leptoquarks" at $250-300 \mathrm{GeV}$ [42].

\subsection{Dark Matter, SUSY Look-alikes and Consistency with Unification}

Vectorlike Confinement models can readily contain dark matter candidates. There are two generic reasons for the existence of extremely long-lived or stable $\tilde{\pi}$. The first possibility is that one (or more) species numbers remain unbroken by nonrenormalizable interactions. A simple example is given by the following model with two species:

\begin{tabular}{c|c||c|c|c} 
& $\mathrm{SU}(N)_{\mathrm{HC}}$ & $\mathrm{SU}(3)_{\mathrm{C}}$ & $\mathrm{SU}(2)_{\mathrm{W}}$ & $\mathrm{U}(1)_{\mathrm{Y}}$ \\
\hline$\Psi_{\mathbf{1}}$ & $\square$ & $\mathbf{1}$ & $\mathbf{1}$ & 1 \\
$\Psi_{\mathbf{3}}$ & $\square$ & $\mathbf{1}$ & $\mathbf{3}$ & 1
\end{tabular}

This model contains, in addition to several $\tilde{\pi}$ of the short-lived type, a long-lived complex $\mathrm{SU}(2)$ triplet hyperpion, with charged and neutral components split by $170 \mathrm{MeV}$ after electroweak symmetry breaking. This complex scalar triplet made out of a $\Psi_{1}$ and a $\Psi_{3}$ (which we will denote as $\tilde{\pi}_{13}$ ) can be stabilized by both an unbroken $\Psi_{\mathbf{1}}$ or an unbroken $\Psi_{\mathbf{3}}$ number 13 . More precisely, the neutral (but complex) component $\tilde{\pi}_{13}^{0}$ is exactly stable, while the two copies of $\tilde{\pi}_{13}^{ \pm}$can decay

\footnotetext{
${ }^{13}$ In an earlier version of this work, the hypercharge of $\Psi_{1,3}$ was assigned to be zero, leading to additional stable bound states due to a mechanism similar to the one described in appendix A We thank Richard Hill for pointing this out to us.
} 
to $\tilde{\pi}_{13}^{0}$ via the SM weak interactions. The $\tilde{\pi}_{13}^{0}$ is therefore a WIMP and a dark matter candidate. When the mass of this WIMP is $\sim 2.0 \mathrm{TeV}$, it has the correct relic density [20]. Since the lightest hyperbaryon in this model (made out of $N_{\mathrm{HC}}$ copies of $\Psi_{1}$ ) is a SM singlet, it is straightforward to write terms that make it decay, for instance to a pair of Higgs bosons when $N_{\mathrm{HC}}$ is even.

The second possibility arises if the lightest long-lived $\tilde{\pi}$ has quantum numbers such that no operator of the form (3.15) or (3.14) can be written down. While such $\tilde{\pi}$ may still decay, this would have to occur through operators with high mass dimension, and lifetimes much larger than the age of the universe become plausible. Such models may therefore contain candidates for decaying dark matter.

One specific possibility we wish to mention is that of a "spectator" species, where one species $\Psi_{\text {spec }}$ lives in an exotic SM representation (e.g. a 4 of $\mathrm{SU}(2)_{\mathrm{W}}$ ). In this case it is possible that various $\tilde{\pi}$ containing $\Psi_{\text {spec }}$ decay into one another, giving rise to (slow) cascades, until the lightest state containing $\Psi_{\text {spec }}$ is reached, which, if neutral, is a dark matter candidate.

To highlight one more interesting phenomenological feature that can arise in Vectorlike Confinement models, consider the following model with 3-species (6-flavors):

\begin{tabular}{c|c||c|c|c} 
& $\mathrm{SU}(N)_{\mathrm{HC}}$ & $\mathrm{SU}(3)_{\mathrm{C}}$ & $\mathrm{SU}(2)_{\mathrm{W}}$ & $\mathrm{U}(1)_{\mathrm{Y}}$ \\
\hline$\Psi_{\mathbf{3}}$ & $\square$ & $\square$ & $\mathbf{1}$ & $-1 / 3$ \\
$\Psi_{\mathbf{2}}$ & $\square$ & $\mathbf{1}$ & $\square$ & $1 / 2$ \\
$\Psi_{\mathbf{1}}$ & $\square$ & $\mathbf{1}$ & $\mathbf{1}$ & 0
\end{tabular}

Note that $\Psi_{\mathbf{1 , 2}, \mathbf{3}}$ are Dirac fermion 14 . One sees that the $\tilde{\pi}$ corresponding to " $\bar{\Psi}_{\mathbf{2}} \Psi_{\mathbf{3}}$ ", " $\bar{\Psi}_{\mathbf{1}} \Psi_{\mathbf{3}}$ " and " $\bar{\Psi}_{1} \Psi_{\mathbf{2}}$ " are like the left-handed squark doublet, right-handed down squark and left-handed slepton doublet (except that the "left-handed squark" has a wrong hypercharge). Their squared-masses from SM gauge loops (3.9) are $\propto g^{2} C_{2}$, resembling a typical sfermion spectrum in gauge-mediated SUSY models. Of course, the collider phenomenology of this model differs significantly from that of typical supersymmetric models, because not only we have a plethora of 4-gauge boson final states but also these "sfermions" will be CHAMPs and R-hadrons, unless extra flavor structures are imposed on the 4 -fermion operators (2.10) and (2.11). If we do impose extra flavor symmetries and allow the "sfermions" to decay at collider time scales, they decay to a pair of SM fermions. Similar features also appear in the "SUSY in slow motion" scenario [43], except for the absence of R-parity.

In fact, since in this model the lightest $\tilde{\pi}$ with the $\bar{\Psi}_{2} \Psi_{1}$ quantum number has a neutral component, one could imagine obtaining a WIMP DM candidate as above by having an unbroken $\Psi_{1}$ species number. However, DM candidates with nonzero hypercharge are strongly constrained by direct detection experiments and at mass scales where this WIMP would have the correct relic abundance, it is excluded by experiment.

Notice also that the quantum numbers of the hyperquarks in this model is consistent with grand unification. The unification of the gauge couplings can be better than that in the SM due to larger threshold corrections enhanced by the hypercolor multiplicity factor $N$.

\footnotetext{
${ }^{14}$ Contrary to our generic assumptions, this particular setup allows the inclusion in the Lagrangian of a Yukawa coupling of $\bar{\Psi}_{2} \Psi_{1}$ to the Higgs doublet. In order to avoid a conflict with precision electroweak constraints we will assume here that such a coupling, if it exists, is small, which is just as natural as taking the mass scale appearing in (3.16) to be large.
} 
Finally, one can write down hyperbaryon number violating operators as in (4.17) and (4.26), for instance in the case of $N_{\mathrm{HC}}=3$ the operator

$$
\mathcal{O}_{\mathrm{HB}}=\epsilon^{A B C}\left(\Psi_{\mathbf{3} A}^{\mathrm{T}} \mathcal{C} \Psi_{\mathbf{3} B}\right)\left(\bar{e}_{\mathrm{R}} \Psi_{\mathbf{3} C}\right)+\text { h.c. }
$$

can be added to the Lagrangian, where $e_{\mathrm{R}}$ is the right-handed electron field in the SM and the $\mathrm{SU}(3)_{\mathrm{C}}$ indices are contracted with an $\epsilon^{i j k}$. For different choices of $N_{\mathrm{HC}}$, one can add insertions of $\Psi_{1}, \Psi_{2}$ or SM fields to make similar operators gauge invariant.

\section{Summary and Conclusions}

We have studied the phenomenology of "Vectorlike Confinement", a broad class of simple confining gauge theories with the confining scales around a TeV. The new matter fields charged under the new gauge group are assumed to be non-chiral under all SM gauge groups, which ensures negligible impact on electroweak precision observables. The quantum numbers of the new matter fields are such that their only renormalizable couplings to the SM are gauge interactions. This guarantees that the models are safe from precision flavor constraints, up to the effect of nonrenormalizable operators which are suppressed by large mass scales.

At hadron colliders, spin-1 bound states of this new confining sector can be resonantly produced via a mechanism exactly analogous to the $\gamma-\rho$ mixing in the resonant production of $\rho$ mesons in $e^{+}-e^{-}$collisions. These new spin-1 bound states can therefore be regarded as a heavy gluon, a heavy $W^{ \pm}$or $Z$, depending on their quantum numbers.

Instead of decaying back to SM particles, these spin-1 resonances dominantly decay to a pair of lighter scalar bound states, analogously to the dominance of the $\rho$ width by $\pi \pi$, which makes the models insensitive to stringent resonance searches in dijets and dileptons. Being pseudo-Goldstone bosons, these scalar bound states are naturally light, in a few hundred $\mathrm{GeV}$ range.

These light scalar bound states exhibit a rich collider phenomenology. They can be classified into two types. The scalars of the first type are analogous to $\pi^{0}$ in the sense that they are made of a particle and an antiparticle of the same species and can decay promptly to a pair of gauge bosons. The scalars of the second type are analogous to $\pi^{ \pm}$in the sense that they are made of constituents of different species and their decays require interactions that can change species. Just as $\pi^{ \pm}$lives much longer than $\pi^{0}$ because changing species (from $u$ to $d$ ) can only occur via nonrenormalizable 4-fermion operators, our scalars of the second type are naturally long-lived.

Therefore, the generic collider signatures of Vectorlike Confinement are the production of four gauge bosons from the decays of a pair of " $\pi^{0}$-type" scalars, and the production of a pair of " $\pi^{ \pm}$type" scalars. The latter is especially interesting because, being made of different species, they typically carry non-trivial SM charges, thereby appearing as CHAMPs or R-hadrons in colliders. An observable fraction of these CHAMPs and R-hadrons are produced from the decays of the aforementioned spin-1 resonances, so the invariant mass distributions of CHAMP pairs and Rhadron pairs exhibit a significant feature hinting at the existence of the parent spin-1 resonance.

In cases where an extra flavor structure in the coefficients of (3.16) allows the mass scale suppressing these operators to be lowered, the lifetimes of " $\pi^{ \pm}$-type" scalars can be short enough to decay inside the collider. In the models we discussed, they preferentially decay to the 3rd generation, analogous to the preference of the $\pi^{ \pm}$decay over muons to electrons. Therefore, depending on their quantum numbers, they can appear as "di- $\tau$," "di-b," "di-top," or "3rd-generation leptoquark." 
We have also discussed the possibility of a spectator species, which occurs in an exotic SM representation and therefore is naturally long-lived even on cosmological time scales, i.e. a dark matter candidate. Heavier states carrying the spectator can cascade decay to lighter ones, which can occur promptly or just slow enough to be visible in the detector. Other models can exhibit SUSY-like spectra of scalars, SUSY-like collider signatures (leptons + jets + missing $E_{\mathrm{T}}$ ) and dark matter candidates, as well as the possibility of accommodating grand unification.

To conclude, a broad class of very simple gauge theories can exhibit extremely rich and exotic collider phenomenology while having virtually no constraints from existing precision measurements. In this sense the spirit is very similar to those of "Quirks" [6] and "Hidden Valley" models [7. Taken altogether, it appears that there is an excellent opportunity for the LHC to discover and explore a new gauge symmetry and its dynamics beyond the SM.

\section{Acknowledgments}

The authors would like to thank Richard Hill, David E. Kaplan, Kirill Melnikov, Matt Strassler, Brock Tweedie and Jay Wacker for useful discussions and insights. C.K. would also like to thank Steffen Schumann for assistance in using Sherpa. The authors are supported by the National Science Foundation grant NSF-PHY-0401513 and by the Johns Hopkins Theoretical Interdisciplinary Physics and Astrophysics Center. C.K. and T.O. are further supported in part by DOE grant DEFG02-03ER4127 and by the Alfred P. Sloan Foundation. T.O. is also supported by the Maryland Center for Fundamental Physics.

\section{Appendices}

\section{A A 2-flavor Model}

In this section we wish to study in detail one of the simplest models of Vectorlike Confinement and illustrate some intriguing theoretical subtleties that it contains. We take only one species of $\Psi$ which is an $\mathrm{SU}(2)$ doublet:

\begin{tabular}{c|c||c|c|c} 
& $\mathrm{SU}(N)_{\mathrm{HC}}$ & $\mathrm{SU}(3)_{\mathrm{C}}$ & $\mathrm{SU}(2)_{\mathrm{W}}$ & $\mathrm{U}(1)_{\mathrm{Y}}$ \\
\hline$\Psi$ & $\square$ & $\mathbf{1}$ & $\square$ & 0
\end{tabular}

Note that $\Psi$ can have no renormalizable couplings to the Higgs or SM fermions, rendering the model robustly safe from precision electroweak and flavor constraints.

The $\tilde{\rho}$ and $\tilde{\pi}$ quantum numbers are

$$
\begin{aligned}
\left(W^{\prime 0}, W^{\prime \pm}\right) & \sim(\mathbf{1}, \mathbf{3})_{0}, \\
\tilde{\rho}^{\prime 0} & \sim(\mathbf{1}, \mathbf{1})_{0}, \\
\left(\tilde{\pi}^{0}, \tilde{\pi}^{ \pm}\right) & \sim(\mathbf{1}, \mathbf{3})_{0} .
\end{aligned}
$$

The $\tilde{\pi}$ mass from $W$ loops before EWSB is given by (3.9):

$$
m_{\tilde{\pi}^{0}, \tilde{\pi}^{ \pm}} \simeq 0.13 c m_{\tilde{\rho}} .
$$

After EWSB, the $\tilde{\pi}^{ \pm}$and $\tilde{\pi}^{0}$ masses split as in (3.11), and the $\tilde{\pi}^{+}$will dominantly decay to $W^{+*} \pi^{0}$. As in the case of Sec. 4.2, the decay product of this off-shell $W^{+}$will be too soft to be detectable. 
Now, naively, one might expect that $\tilde{\pi}^{0}$ would decay promptly to weak gauge bosons via the vertex (3.1d). This vertex, however, vanishes for $\mathrm{SU}(2)$, and in fact there is a $\mathbb{Z}_{2}$ symmetry which prevents a $\tilde{\pi}^{0}$ from decaying. This $\mathbb{Z}_{2}$ symmetry is an accidental symmetry of the renormalizable Lagrangian under which the hypercolor gauge field $H_{\mu}$ and the hyperquarks $\psi$ and $\psi^{\mathrm{c}}$ transform as

$$
\begin{aligned}
\Psi & \longrightarrow i \tau_{2} \mathcal{C} \Psi^{*}, \\
H_{\mu} & \longrightarrow-H_{\mu}^{\mathrm{T}},
\end{aligned}
$$

where the Pauli matrix $\tau_{2}$ acts on the $\mathrm{SU}(2)_{\mathrm{W}}$ indices and $\mathcal{C}$ is the charge conjugation matrix. All SM fields are invariant under this $\mathbb{Z}_{2}$. The $\langle\bar{\Psi} \Psi\rangle$ condensate respects the symmetry upon confinement, so the $\mathbb{Z}_{2}$ is not spontaneously broken. Note that a $\mathbb{Z}_{2}$ symmetry of this kind exists if and only if all hyperquarks are in (pseudo)real SM gauge representations (zero hypercharge and e.g. an adjoint of $\left.\mathrm{SU}(3)_{\mathrm{C}}\right)$. Such $\mathbb{Z}_{2}$ symmetries are studied extensively in [44].

Now, note that the $\tilde{\pi}$ is odd under the $\mathbb{Z}_{2}($ A.3 $)$, because the hyper-axial current

$$
J_{5}^{a \mu} \equiv \bar{\Psi} \frac{\tau^{a}}{2} \gamma_{5} \gamma^{\mu} \Psi \quad(a=1,2,3)
$$

is odd. Therefore, in the absence of nonrenormalizable terms in the Lagrangian, the $\mathbb{Z}_{2}$ renders the $\tilde{\pi}^{0}$ stable.

The $\mathbb{Z}_{2}$ symmetry is sensitive to non-renormalizable operators, but it is interesting to contemplate whether it can be imposed exactly as the $\tilde{\pi}^{0}$ can be a good dark matter candidate. The problem is that the fate of the $\tilde{\pi}^{0}$ and the hyperbaryons are connected. Note that in this model the hyperbaryons will have half-integer electric charge if the number of hypercolor $N$ is odd, while they will be SM singlets if $N$ is even. The lightest hyperbaryon in the former case would be absolutely stable due to its fractional electric charge, which is clearly excluded by observation. In the latter case, the choice of $\Lambda_{H C}$ for which $\tilde{\pi}^{0}$ has the correct relic density also leads to the lightest hyperbaryon to have roughly the correct relic density. Without performing a more sophisticated analysis, it is not clear which will be the dominant dark matter component, therefore drawing quantitative conclusions goes beyond the scope of this paper. On the other hand, attempting to break hyperbaryon number generically also breaks the $\mathbb{Z}_{2}$ symmetry, thereby rendering the $\tilde{\pi}^{0}$ unstable, such that one loses both dark matter candidates rather than only one of them.

With the $\mathbb{Z}_{2}$ broken, the $\tilde{\pi}^{0}$ will either decay to gauge boson pairs or SM fermions, depending on which type of nonrenormalizable operator dominates. The phenomenology of such decays is discussed in the model presented in Sec. 4.2 where they happen generically, while in this model they would only occur through additional $\mathbb{Z}_{2}$-violating terms. For this reason we choose not to elaborate on the phenomenology of this model as a representative case.

\section{B Collider Constraints on Axion-like $\tilde{\pi}$}

If an axion has a coupling to gluons as well as photons, there is a potential constraint on $\tilde{\pi}_{0}^{\prime}$ production at hadron colliders with subsequent decays to a pair of photons. We use the measurement presented in [41] to check whether the predicted diphoton cross section is consistent with experiment. The simplest production mechanism is $g g \rightarrow \tilde{\pi}_{0}^{\prime}$; while the production rate is loop suppressed due to (3.1d) and the branching fraction $\tilde{\pi}_{0}^{\prime} \rightarrow \gamma \gamma$ is suppressed by $\left(\alpha_{\mathrm{EM}} / \alpha_{\mathrm{s}}\right)^{2}$, the gluon PDF's at low energy are large enough to give a sizeable cross section for this process. 


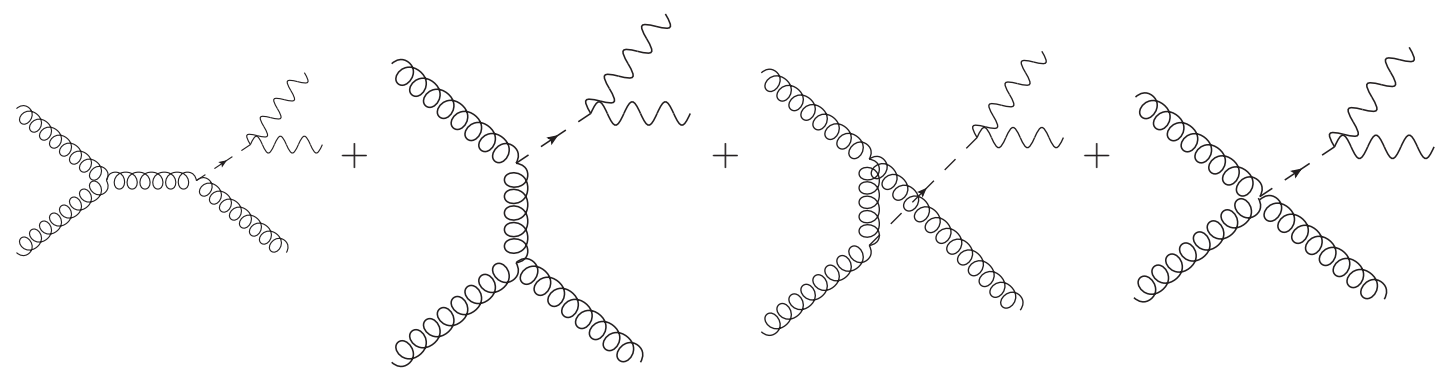

Figure 6: The Feynman diagrams contributing to the $g g \rightarrow \tilde{\pi}_{0}^{\prime} g$ process.

However, when a $\tilde{\pi}_{0}^{\prime}$ is produced this way, it has zero transverse momentum, and the photons from the decay will not satisfy the $p_{\mathrm{T}}$ cuts used in [41] unless $m_{\tilde{\pi}_{0}^{\prime}} \gtrsim 30 \mathrm{GeV}$. Of course, this is an oversimplification, since there will always be some amount of gluon radiation in the initial state, which the axion can recoil against. In the case that this radiation is hard, one can think of it as the $2 \rightarrow 2$ process illustrated in figure Fig. 6. However, the amplitude for this process has a singularity and therefore cannot be used for the region of soft radiation. Thus, in order to study whether [41] rules out the existence of a $\tilde{\pi}_{0}^{\prime}$, one needs to analyze a matched sample of $g g \rightarrow \tilde{\pi}_{0}^{\prime}+$ jet(s) events, which interpolates between the $2 \rightarrow 1$ approximation for heavy axions and the $2 \rightarrow 2$ approximation for light axions.

To accomplish this, we have generated a matched event sample using SHERPA 1.1 [45] with ISR turned on. Implementing the experimental cuts used in [41, we find that the existence of $\tilde{\pi}_{0}^{\prime}$ is consistent with data for the reference values of $f_{\tilde{\pi}}=200 \mathrm{GeV}$ and the number of hypercolor $N=3$, for all $m_{\tilde{\pi}_{0}^{\prime}}$.

While there are also contributions to axion production from $q g \rightarrow \tilde{\pi}_{0}^{\prime}+$ jet(s) and from $q \bar{q} \rightarrow$ $\tilde{\pi}_{0}^{\prime}+$ jet(s), we find that these give cross sections much smaller than $g g \rightarrow \tilde{\pi}_{0}^{\prime}+$ jet(s).

Therefore, we conclude that there are no relevant constraints from hadron colliders on the existence of axions in our model heavier than $100 \mathrm{MeV}$.

\section{References}

[1] N. Arkani-Hamed and S. Dimopoulos, "Supersymmetric unification without low energy supersymmetry and signatures for fine-tuning at the LHC," JHEP 0506, 073 (2005) arXiv:hep-th/0405159.

[2] G. D. Kribs, T. Plehn, M. Spannowsky and T. M. P. Tait, "Four generations and Higgs physics," Phys. Rev. D 76, 075016 (2007) [arXiv:0706.3718 [hep-ph]].

[3] E. Eichten and K. D. Lane, "Dynamical Breaking Of Weak Interaction Symmetries," Phys. Lett. B 90, 125 (1980).

[4] C. Kilic, T. Okui and R. Sundrum, "Colored Resonances at the Tevatron: Phenomenology and Discovery Potential in Multijets," JHEP 0807, 038 (2008) [arXiv:0802.2568 [hep-ph]].

[5] C. Kilic, S. Schumann and M. Son, "Searching for Multijet Resonances at the LHC," JHEP 0904, 128 (2008) arXiv:0810.5542 [hep-ph]]. 
[6] J. Kang and M. A. Luty, "Macroscopic Strings and 'Quirks' at Colliders," arXiv:0805.4642 [hep-ph].

[7] M. J. Strassler and K. M. Zurek, "Echoes of a hidden valley at hadron colliders," Phys. Lett. B 651, 374 (2007) arXiv:hep-ph/0604261]; T. Han, Z. Si, K. M. Zurek and M. J. Strassler, "Phenomenology of Hidden Valleys at Hadron Colliders," JHEP 0807, 008 (2008) arXiv:0712.2041 [hep-ph]].

[8] L. Susskind, "Dynamics Of Spontaneous Symmetry Breaking In The Weinberg-Salam Theory," Phys. Rev. D 20, 2619 (1979); S. Weinberg, "Implications Of Dynamical Symmetry Breaking," Phys. Rev. D 13, 974 (1976); S. Weinberg, "Implications Of Dynamical Symmetry Breaking: An Addendum," Phys. Rev. D 19, 1277 (1979).

[9] E. Farhi and L. Susskind, "Technicolor," Phys. Rept. 74, 277 (1981); C. T. Hill and E. H. Simmons, "Strong dynamics and electroweak symmetry breaking," Phys. Rept. 381, 235 (2003) [Erratum-ibid. 390, 553 (2004)] arXiv:hep-ph/0203079].

[10] K. Lane and S. Mrenna, "The Collider phenomenology of technihadrons in the technicolor straw man model," Phys. Rev. D 67, 115011 (2003) arXiv:hep-ph/0210299.

[11] G. H. Brooijmans et al., "New Physics at the LHC: A Les Houches Report. Physics at Tev Colliders 2007 - New Physics Working Group," arXiv:0802.3715 [hep-ph].

[12] F. Sannino, "Dynamical Stabilization of the Fermi Scale: Phase Diagram of Strongly Coupled Theories for (Minimal) Walking Technicolor and Unparticles," arXiv:0804.0182 [hep-ph].

[13] T. W. Appelquist, D. Karabali and L. C. R. Wijewardhana, "Chiral Hierarchies and the Flavor Changing Neutral Current Problem in Technicolor," Phys. Rev. Lett. 57, 957 (1986); T. Appelquist and L. C. R. Wijewardhana, "Chiral Hierarchies And Chiral Perturbations In Technicolor," Phys. Rev. D 35, 774 (1987).

[14] M. A. Luty and T. Okui, "Conformal technicolor," JHEP 0609, 070 (2006) arXiv:hep-ph/0409274]; M. A. Luty, "Strong Conformal Dynamics at the LHC and on the Lattice," JHEP 0904, 050 (2009) [arXiv:0806.1235 [hep-ph]].

[15] D. D. Dietrich, F. Sannino and K. Tuominen, "Light composite Higgs from higher representations versus electroweak precision measurements: Predictions for LHC," Phys. Rev. D 72, 055001 (2005) arXiv:hep-ph/0505059]; R. Foadi, M. T. Frandsen, T. A. Ryttov and F. Sannino, "Minimal Walking Technicolor: Set Up for Collider Physics," Phys. Rev. D 76, 055005 (2007) arXiv:0706.1696 [hep-ph]]; E. Eichten and K. Lane, "Low-scale technicolor at the Tevatron and LHC," Phys. Lett. B 669, 235 (2008) arXiv:0706.2339 [hep-ph]]; A. Belyaev, R. Foadi, M. T. Frandsen, M. Jarvinen, F. Sannino and A. Pukhov, "Technicolor Walks at the LHC," Phys. Rev. D 79, 035006 (2009) [arXiv:0809.0793 [hep-ph]].

[16] C. Kilic and T. Okui, in preparation.

[17] B. Holdom and J. Terning, "Large Corrections To Electroweak Parameters In Technicolor Theories," Phys. Lett. B 247, 88 (1990); M. Golden and L. Randall, "Radiative Corrections To Electroweak Parameters In Technicolor Theories," Nucl. Phys. B 361, 3 (1991).

[18] M. E. Peskin and T. Takeuchi, "Estimation of oblique electroweak corrections," Phys. Rev. D 46, 381 (1992). 
[19] M. Bona et al. [UTfit Collaboration], "Model-independent constraints on $\Delta \mathrm{F}=2$ operators and the scale of new physics," JHEP 0803, 049 (2008) [arXiv:0707.0636 [hep-ph]].

[20] M. Cirelli, N. Fornengo and A. Strumia, "Minimal dark matter," Nucl. Phys. B 753, 178 (2006) arXiv:hep-ph/0512090.

[21] C. Amsler et al. [Particle Data Group], "Review of particle physics," Phys. Lett. B 667, 1 (2008) and references therein.

[22] V. M. Abazov et al. [D0 Collaboration], "Search for a scalar or vector particle decaying into $Z \gamma$ in $p \bar{p}$ collisions at $\sqrt{s}=1.96-\mathrm{TeV}$," arXiv:0806.0611 [hep-ex].

[23] V. M. Abazov et al. [D0 Collaboration], "Search for long-lived particles decaying into electron or photon pairs with the D0 detector," Phys. Rev. Lett. 101, 111802 (2008) arXiv:0806.2223 [hep-ex]].

[24] A. L. Scott [CDF Collaboration and representing the CDF Collaboration], "Search for longlived parents of the Z0 boson," Int. J. Mod. Phys. A 20, 3263 (2005) arXiv:hep-ex/0410019.

[25] P. F. Smith and J. R. J. Bennett, "A Search For Heavy Stable Particles," Nucl. Phys. B 149, 525 (1979).

[26] T. K. Hemmick et al., "A SEARCH FOR ANOMALOUSLY HEAVY ISOTOPES OF LOW Z NUCLEI," Phys. Rev. D 41, 2074 (1990).

[27] P. Verkerk, G. Grynberg, B. Pichard, M. Spiro, S. Zylberajch, M. E. Goldberg and P. Fayet, "Search for superheavy hydrogen in sea water," Phys. Rev. Lett. 68, 1116 (1992).

[28] T. Yamagata, Y. Takamori and H. Utsunomiya, "Search for anomalously heavy hydrogen in deep sea water at 4000-m," Phys. Rev. D 47, 1231 (1993).

[29] P. F. Smith, J. R. J. Bennett, G. J. Homer, J. D. Lewin, H. E. Walford and W. A. Smith, "A Search For Anomalous Hydrogen In Enriched D-2 O, Using A Time-Of-Flight Spectrometer," Nucl. Phys. B 206, 333 (1982).

[30] K. Hatakeyama [CDF Collaboration], "High Mass Resonance Searches at CDF," arXiv:0810.3681 [hep-ex].

[31] CDF Collaboration, CDF public note 9160.

[32] T. Aaltonen et al. [The CDF Collaboration], "A search for high-mass resonances decaying to dimuons at CDF," arXiv:0811.0053 [hep-ex].

[33] D0 Collaboration, D0 public note 4375.

[34] D0 Collaboration, D0 public note 4577.

[35] F. Bergsma et al. [CHARM Collaboration], "Search For Axion Like Particle Production In 400-GeV Proton - Copper Interactions," Phys. Lett. B 157, 458 (1985); E. M. Riordan et al., "A Search For Short Lived Axions In An Electron Beam Dump Experiment," Phys. Rev. Lett. 59, 755 (1987); J. D. Bjorken et al., "Search for Neutral Metastable Penetrating Particles Produced in the SLAC Beam Dump," Phys. Rev. D 38, 3375 (1988); A. Bross, M. Crisler, S. H. Pordes, J. Volk, S. Errede and J. Wrbanek, "A Search For Shortlived Particles Produced In An Electron Beam Dump," Phys. Rev. Lett. 67, 2942 (1991).

[36] J. D. Bjorken, R. Essig, P. Schuster and N. Toro, "New Fixed-Target Experiments to Search for Dark Gauge Forces," Phys. Rev. D 80, 075018 (2009) [arXiv:0906.0580 [hep-ph]]. 
[37] B. Aubert et al. [BaBar Collaboration], "Search for Invisible Decays of a Light Scalar in Radiative Transitions $v_{3 S} \rightarrow \gamma$ A0," arXiv:0808.0017 [hep-ex].

[38] A. Rosca [LEP Collaborations], "Fermiophobic Higgs bosons at LEP," arXiv:hep-ex/0212038.

[39] V. M. Abazov et al. [D0 Collaboration], "Search for Long-Lived Charged Massive Particles with the D0 Detector," Phys. Rev. Lett. 102, 161802 (2009) [arXiv:0809.4472 [hep-ex]].

[40] T. Aaltonen et al. [CDF Collaboration], "Search for Long-Lived Massive Charged Particles in $1.96 \mathrm{TeV} \bar{p} p$ Collisions," Phys. Rev. Lett. 103, 021802 (2009) [arXiv:0902.1266 [hep-ex]].

[41] D. E. Acosta et al. [CDF Collaboration], "Measurement of the cross section for prompt diphoton production in $p \bar{p}$ collisions at $\sqrt{s}=1.96 \mathrm{TeV}, "$ Phys. Rev. Lett. 95, 022003 (2005) arXiv:hep-ex/0412050.

[42] V. M. Abazov et al. [D0 Collaboration], "Search for third-generation leptoquarks in $p \bar{p}$ collisions at $\sqrt{s}=1.96-\mathrm{TeV}, "$ Phys. Rev. Lett. 99, 061801 (2007) arXiv:0705.0812 [hep-ex]]; V. M. Abazov et al. [D0 Collaboration], "Search for third generation scalar leptoquarks decaying into $\tau b, "$ arXiv:0806.3527 [hep-ex].

[43] Z. Chacko, C. A. Krenke and T. Okui, "Supersymmetry in Slow Motion," arXiv:0809.3820 [hep-ph].

[44] Y. Bai and R. J. Hill, "Weakly Interacting Stable Pions," arXiv:1005.0008 [hep-ph].

[45] T. Gleisberg, S. Hoche, F. Krauss, M. Schonherr, S. Schumann, F. Siegert and J. Winter, "Event generation with SHERPA 1.1," JHEP 0902, 007 (2009) arXiv:0811.4622 [hep-ph]]. 